Roger Williams University

DOCS@RWU

2013

\title{
GRB 091024A AND THE NATURE OF ULTRA-LONG GAMMA-RAY BURSTS
}

\author{
F.J. Virgili \\ C.G. Mundell \\ V. Pal'shin \\ C. Guidorzi \\ R. Margutti
}

See next page for additional authors

Follow this and additional works at: https://docs.rwu.edu/fcas_fp

Part of the Astrophysics and Astronomy Commons

\section{Recommended Citation}

Virgil. F.J. ...... A. C. Updike, et al. "GRB 091024A and the Nature of Ultra-Long GRBs." Astrophysical Journal Volume 778 (2013):54

This Article is brought to you for free and open access by the Arts and Sciences at DOCS@RWU. It has been accepted for inclusion in Arts \& Sciences Faculty Publications by an authorized administrator of DOCS@RWU. For more information, please contact mwu@rwu.edu. 


\section{Authors}

F.J. Virgili, C.G. Mundell, V. Pal'shin, C. Guidorzi, R. Margutti, A. Melandri, R. Harrison, S. Kobayashi, R. Chornock, A. Henden, Adria Updike, S.B. Cenko, N.R. Tanvir, I.A. Steele, A. Cucchiara, A. Gomboc, A. Levan, Z. Cano, C.J. Mottram, N.R. Clay, D. Bersier, D. Kopa`c, J. Japelj, A.V. Filippenko, W. Li, D. Svinkin, S. Golenetskii, D.H. Hartmann, P.A. Milne, G. Williams, P.T. O’Brien, D.B. Fox, and E. Berger 


\title{
GRB 091024A AND THE NATURE OF ULTRA-LONG GAMMA-RAY BURSTS
}

\author{
F. J. Virgili ${ }^{1}$, C. G. Mundell ${ }^{1}$, V. Pal'shin ${ }^{2}$, C. Guidorzi ${ }^{3}$, R. Margutti ${ }^{4}$, A. Melandri ${ }^{5}$, R. Harrison ${ }^{1}$, S. Kobayashi $^{1}$,

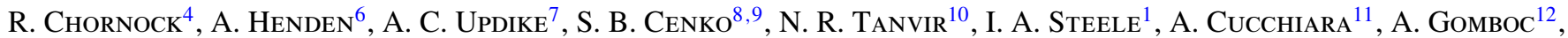 \\ A. Levan $^{13}$, Z. Cano ${ }^{14}$, C. J. Mottram ${ }^{1}$, N. R. Clay ${ }^{1}$, D. Bersier ${ }^{1}$, D. KopaČ ${ }^{12}$, J. Japelj $^{12}$, A. V. Filippenko ${ }^{8}$, \\ W. Li ${ }^{8,18}$, D. Svinkin ${ }^{2}$, S. GolenetskiI ${ }^{2}$, D. H. Hartmann ${ }^{15}$, P. A. Milne ${ }^{16}$, G. Williams ${ }^{16}$, \\ P. T. O'BRIEN ${ }^{10}$, D. B. Fox ${ }^{17}$, AND E. BERGER ${ }^{4}$ \\ ${ }^{1}$ Astrophysics Research Institute, Liverpool John Moores University, Liverpool, L3 5RF, UK; F.J.Virgili@ljmu.ac.uk \\ ${ }^{2}$ Ioffe Physical Technical Institute, St. Petersburg 194021, Russia \\ ${ }^{3}$ Department of Physics and Earth Sciences, University of Ferrara, Via Saragat, 1, I-44122 Ferrara, Italy \\ ${ }^{4}$ Harvard-Smithsonian Center for Astrophysics, 60 Garden Street, Cambridge, MA 02138, USA \\ ${ }^{5}$ INAF/Brera Astronomical Observatory, via Bianchi 46, I-23807 Merate (LC), Italy \\ ${ }^{6}$ AAVSO, 49 Bay State Road, Cambridge, MA 02138, USA \\ ${ }^{7}$ Department of Chemistry and Physics, Roger Williams University, Bristol, RI 02809, USA \\ 8 Department of Astronomy, University of California, Berkeley, CA 94720-3411, USA \\ ${ }^{9}$ Astrophysics Science Division, NASA Goddard Space Flight Center, Mail Code 661, Greenbelt, MD 20771, USA \\ ${ }^{10}$ Department of Physics and Astronomy, University of Leicester, University Road, Leicester LE1 7RH, UK \\ ${ }^{11}$ Department of Astronomy and Astrophysics, UCO/Lick Observatory, University of California, 1156 High Street, Santa Cruz, CA 95064, USA \\ ${ }^{12}$ Faculty of Mathematics and Physics, University of Ljubljana, Jadranska 19, 1000 Ljubljana, Slovenia \\ ${ }^{13}$ Department of Physics, University of Warwick, Coventry CV4 7AL, UK \\ ${ }^{14}$ Centre for Astrophysics and Cosmology, Science Institute, University of Iceland, 107 Reykjavik, Iceland \\ ${ }^{15}$ Department of Physics and Astronomy, 118 Kinard Laboratory, Clemson University, Clemson, SC 29631-0978, USA \\ ${ }^{16}$ MMT Observatory, University of Arizona, Tucson, AZ 85719, USA \\ ${ }^{17}$ Department of Astronomy and Astrophysics, The Pennsylvania State University, 525 Davey Lab, University Park, PA 16802, USA \\ Received 2013 July 1; accepted 2013 September 30; published 2013 November 1
}

\begin{abstract}
We present a broadband study of gamma-ray burst (GRB) 091024A within the context of other ultra-long-duration GRBs. An unusually long burst detected by Konus-Wind (KW), Swift, and Fermi, GRB 091024A has prompt emission episodes covering $\sim 1300 \mathrm{~s}$, accompanied by bright and highly structured optical emission captured by various rapid-response facilities, including the $2 \mathrm{~m}$ autonomous robotic Faulkes North and Liverpool Telescopes, KAIT, S-LOTIS, and the Sonoita Research Observatory. We also observed the burst with 8 and 10 m class telescopes and determine the redshift to be $z=1.0924 \pm 0.0004$. We find no correlation between the optical and $\gamma$-ray peaks and interpret the optical light curve as being of external origin, caused by the reverse and forward shock of a highly magnetized jet $\left(R_{B} \approx 100-200\right)$. Low-level emission is detected throughout the near-background quiescent period between the first two emission episodes of the KW data, suggesting continued central-engine activity; we discuss the implications of this ongoing emission and its impact on the afterglow evolution and predictions. We summarize the varied sample of historical GRBs with exceptionally long durations in gamma-rays ( $\gtrsim 1000$ s) and discuss the likelihood of these events being from a separate population; we suggest ultra-long GRBs represent the tail of the duration distribution of the long GRB population.
\end{abstract}

Key words: gamma-ray burst: general - gamma-ray burst: individual (GRB 091024A)

Online-only material: color figures

\section{INTRODUCTION}

Following the first detection of a gamma-ray burst (GRB) by a military satellite in the late 1960s (Klebesadel et al. 1973), the BATSE $\gamma$-ray detector (Fishman et al. 1993) on board the Compton Gamma-Ray Observatory (CGRO) revolutionized the study of $\gamma$-ray properties, detecting flashes with durations from $t<64 \mathrm{~ms}$ to $t>500 \mathrm{~s}$, showing their sky distribution to be isotropic, and producing a catalog of 1637 GRB light curves (revised 4B catalog; Paciesas et al. 1999). Most notably, GRB $t_{90}$ duration-defined as the time in which 5\%-95\% of the burst fluence is accumulated-has played a key role in GRB classification (Kouveliotou et al. 1993). Initially seen as a powerful discriminator between possible GRB progenitor models, $t_{90}$ has been shown to be sensitive to detector energy range (Sakamoto et al. 2011; Virgili et al. 2012; Qin et al.

\footnotetext{
${ }^{18}$ Deceased 2011 December 11.
}

2013), thus requiring a more complete approach to progenitor categorization (e.g., Zhang et al. 2009a) and the study of emission mechanisms.

GRB 091024A falls into a category of bursts with observed $\gamma$-ray emission lasting longer than $\sim 1000 \mathrm{~s}$. Such emission may be continuous or consist of shorter $(\sim 50-250 \mathrm{~s})$ episodes separated by significant periods of low-level emission or quiescence, complicating the definition of "duration" and the interpretation of its physical significance for different bursts. In some cases, long-lasting emission has been attributed to ongoing central-engine activity. Observational evidence to support this hypothesis has been seen at longer wavelengths in the form of X-ray (Burrows et al. 2005; Zhang et al. 2006; Fan \& Wei 2005; Chincarini et al. 2010; Margutti et al. 2011) and optical flares (Vestrand et al. 2005; Boër et al. 2006; Wei et al. 2006; Melandri et al. 2009) that show characteristics, such as shorttimescale variability, steep rise and decay slopes, and a clear lag-luminosity relation (Margutti et al. 2010), that are, in some 
cases, difficult to reconcile within the standard fireball model or an external-shock origin (Melandri et al. 2010; Kopač et al. 2013). Further support comes from long-lasting X-ray emission prior to the steep decay phase of the X-ray light curve (Zhang et al. 2006), interpreted as curvature radiation from the cessation of central-engine activity (Zhang et al. 2006; Liang et al. 2006a; Yamazaki et al. 2006; Zhang et al. 2009b), and long-duration X-ray-rich bursts (Feroci et al. 2001; Nicastro et al. 2004; in't Zand et al. 2004).

A small number of BATSE bursts were detected with prompt emission lasting $>500 \mathrm{~s}$ and up to $1300 \mathrm{~s}$, but their poor localizations and the resultant lack of multi-wavelength counterparts limit understanding of their nature and origin. The launch of NASA's Swift satellite (Gehrels et al. 2004) with its optimized GRB detection and rapid dissemination of accurate localizations, coupled with real-time follow-up observations by autonomous robotic optical telescopes such as the $2 \mathrm{~m}$ Liverpool and Faulkes telescopes (Guidorzi et al. 2006b) and smaller very rapid-response facilities such as the Katzman Automatic Imaging Telescope ${ }^{19}$ (KAIT; Filippenko et al. 2001; Li et al. 2003), Super-LOTIS (S-LOTIS; Park et al. 1997, 2002), and the Sonoita Research Observatory (SRO), ${ }^{20}$ has opened a new era of multi-wavelength study of GRB properties at early times.

Although ultra-long GRBs remain rare, detection and comprehensive follow-up observations of ultra-long events such as GRBs 091024A, 110709B (Zhang et al. 2012), and 111209A (Gendre et al. 2013; Stratta et al. 2013) are providing new insights into the physics of this extreme subset. These bursts are an interesting laboratory in which to test the framework of the internal/external-shock model and our assumptions of centralengine activity, most notably in the context of accretion onto a black hole (BH) from a very large star (e.g., Gendre et al. 2013; Levan et al 2013, Stratta et al. 2013).

In this paper, we present a detailed analysis of the $\gamma$-ray, X-ray, and optical emission from GRB 091024A, whose observed prompt emission lasted for $\gtrsim 1200 \mathrm{~s}$, allowing simultaneous multi-wavelength observations to be obtained. We show that the optical light curve is consistent with an external-shock origin, and that there is significant $\gamma$-ray emission detected in the period of apparent quiescence between the first two episodes of emission, which has a measurable impact on the observed optical light curve at early times. We place GRB 091024A into a wider context by comparing its properties with a sample of ultra-long GRBs with duration $\gtrsim 1000 \mathrm{~s}$ and discuss whether they represent a new emerging class of GRBs.

The multi-wavelength observations of GRB 091024A are presented in Section 2, and the temporal and spectral analysis together with the derived energetics are presented in Section 3. Section 4 is dedicated to modeling the afterglow. In Section 5 we introduce the sample of historic ultra-long GRBs, discussing their individual properties as members of two classes-continuous and intermittent prompt $\gamma$-ray emission. Section 6 presents a discussion and Section 7 our conclusions. Details of each individual burst in our sample are summarized in the Appendix. Throughout the paper, we use the following conventions: UT dates are used and are relative to the Swift/BAT trigger at $T_{0}=08: 56: 01$ on 2009 October $24 ; F(v, t) \propto t^{-\alpha} v^{-\beta}$; the following cosmological model is used: $H_{0}=71 \mathrm{~km} \mathrm{~s}^{-1} \mathrm{Mpc}$, $\Omega_{m}=0.3, \Omega_{\Lambda}=0.7$; uncertainties are quoted at $1 \sigma$ unless otherwise specified.

\footnotetext{
19 http://astro.berkeley.edu/bait/public_html/kait.html.

$20 \mathrm{http} / / /$ www.aavso.org/sonoita-research-observatory-sro.
}

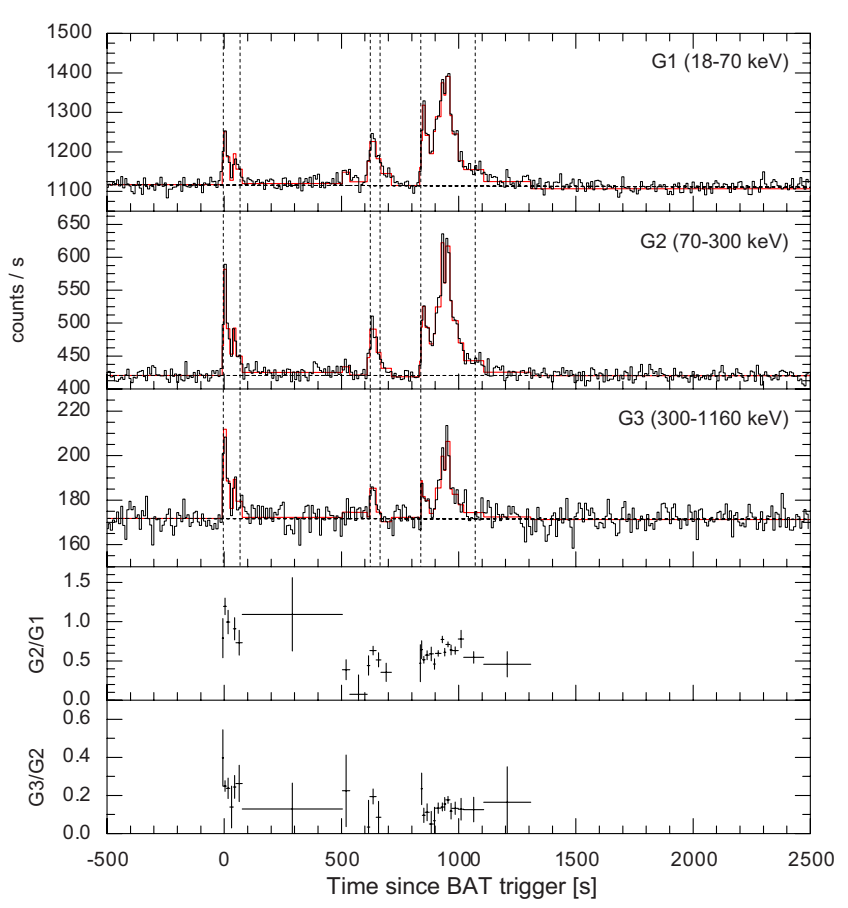

Figure 1. Hardness ratios and light curves of GRB 091024A in the three Konus-Wind bands. Dashed lines are approximate Fermi/GBM durations, highlighting the slight underestimation of the durations of the second and third emission episodes.

(A color version of this figure is available in the online journal.)

\section{OBSERVATIONS}

\subsection{Gamma-Ray and X-Ray Observations}

GRB 091024A was detected at high energies by Konus-Wind (KW; Golenetskii et al. 2009), Swift-Burst Alert Telescope (BAT; Marshall et al. 2009) and X-ray Telescope (XRT; Page \& Marshall 2009), Fermi-Gamma-ray Burst Monitor (GBM; Bissaldi \& Connaughton 2009), and SPI-ACS (Gruber et al. 2011). The data from KW and GBM cover the entire burst duration, with GBM having triggered a second time on the major outburst about $600 \mathrm{~s}$ after the first trigger (Gruber et al. 2011). The KW light curve in Figure 1 shows three multi-peak emission episodes: the first with duration $\delta t \approx 88 \mathrm{~s}$ at $T+T_{0, \mathrm{KW}}=-8.9 \mathrm{~s}$, the second with $\delta t \approx 106 \mathrm{~s}$ at $T+T_{0, \mathrm{KW}}=609 \mathrm{~s}$, and the third with $\delta t \approx 477 \mathrm{~s}$ at $T+T_{0, \mathrm{KW}}=883 \mathrm{~s}$ (with respect to the $\mathrm{KW}$ trigger time; Swift $T_{0}-\mathrm{KW} T_{0} \approx 0.3 \mathrm{~s}$ ).

Swift-BAT and XRT have partial datasets which are truncated because of Earth-limb constraints. The former contains the first emission episode (Figure 2) and the latter emission from $T_{0}+53$ minutes (Page \& Marshall 2009) to $T_{0}+1398$ minutes (Figure 3 ). We processed the BAT data with standard HEAsoft tools ( $v$ 6.10) and utilize the spectra for a joint analysis with the first peak of the KW data.

Although truncated, the XRT X-ray light curve can be adequately fit with a simple power law having a decay slope $\alpha=1.7 \pm 0.1$, following the procedure of Margutti et al. (2013a). The X-ray spectrum can be fit with a simple power law of slope $\Gamma_{X}=1.49_{-0.21}^{+0.23}$, with total column density $N_{\mathrm{H}}=$ $1.7_{-1.1}^{+1.2} \times 10^{22} \mathrm{~cm}^{-2}$ and intrinsic $N_{\mathrm{H}}=3.0_{-1.5}^{+1.7} \times 10^{22} \mathrm{~cm}^{-2}$ (Evans et al. 2007, 2009; Kalberla et al. 2005).

\subsection{Optical Observations}

A number of rapid-response optical facilities, with apertures ranging from $0.35 \mathrm{~m}$ to $2 \mathrm{~m}$, responded to the Swift-BAT trigger 


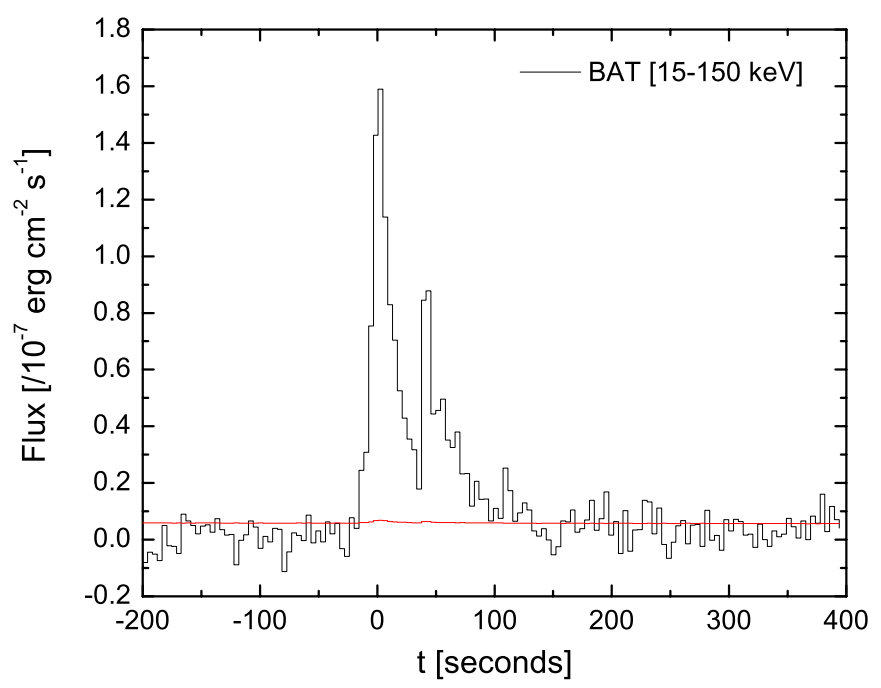

Figure 2. Swift-BAT $4 \mathrm{~s}$ binned light curves $(15-150 \mathrm{keV})$ of the first emission episode $\left(T_{0}<450 \mathrm{~s}\right)$ of GRB 091024A. The remaining $\gamma$-ray emission was observed but no coded-mask information is available.

(A color version of this figure is available in the online journal.)

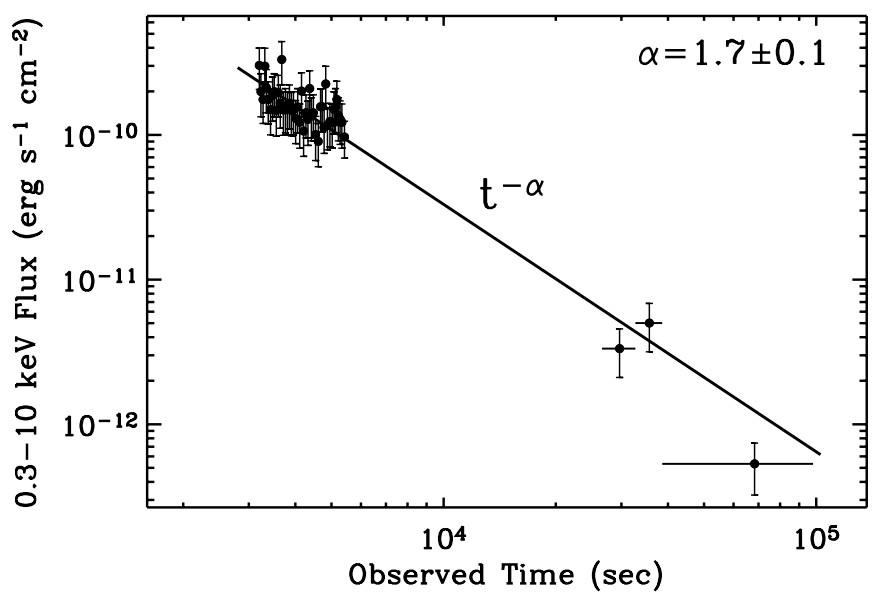

Figure 3. Swift-XRT light curve with a simple power law fit overlaid $(\alpha=$ $1.7 \pm 0.1$ ). Observations began at $T=T_{0}+53$ minutes.

beginning at $T_{0}+58 \mathrm{~s}$. Optical monitoring continued to $T_{0}+10^{6} \mathrm{~s}$, along with redshift determinations by 8 to $10 \mathrm{~m}$ class telescopes. We cross-calibrated our photometric dataset with respect to a common set of standard stars observed in $B V R I$ filters with the SRO telescope during the week after the burst event. Final calibrated magnitudes and extinction-corrected fluxes are summarized in Table 1 and Figure 4.

\subsubsection{Super-LOTIS}

The $0.6 \mathrm{~m} \mathrm{~S}$-LOTIS telescope began observing at $T_{0}+58 \mathrm{~s}$ for a total of 36 minutes (Updike et al. 2009). We began with a series of $5 \times 10 \mathrm{~s}$ exposures, then a sequence of $5 \times 20 \mathrm{~s}$ exposures, and finally increasing to $60 \mathrm{~s}$ exposures after 5 minutes. We grouped and co-added several frames during the observing interval in order to increase the total signal-to-noise ratio $(\mathrm{S} / \mathrm{N})$, and we calibrated these images with respect to the $R$ band.

\subsubsection{Katzman Automatic Imaging Telescope}

The $0.76 \mathrm{~m}$ KAIT began observations at $T_{0}+82 \mathrm{~s}$ (Chornock et al. 2009) with $20 \mathrm{~s}$ exposures in alternating $V$, $I$, and unfiltered bands. Observations ended $\sim 16$ minutes after the trigger. $V$-band and $I$-band images have been calibrated with respect
Table 1

Cross-calibrated and Extinction-corrected Photometry of GRB $091024^{\mathrm{a}}$

\begin{tabular}{|c|c|c|c|c|c|}
\hline $\begin{array}{l}\Delta t^{\mathrm{b}} \\
(\mathrm{s})\end{array}$ & $\begin{array}{c}\text { Exposure } \\
\text { (s) }\end{array}$ & Filter & $\begin{array}{l}\text { Flux }^{c} \\
(m J y)\end{array}$ & Magnitude $^{c}$ & Telescope \\
\hline 440 & 10 & $B$ & $4.35 \pm 0.76$ & $14.96 \pm 0.19$ & FTN \\
\hline 757.2 & 30 & $B$ & $2.18 \pm 0.28$ & $15.71 \pm 0.14$ & FTN \\
\hline 1316 & 60 & $B$ & $1.41 \pm 0.25$ & $16.18 \pm 0.19$ & FTN \\
\hline 2164 & 120 & $B$ & $1.90 \pm 0.18$ & $15.86 \pm 0.1$ & FTN \\
\hline 3260 & 180 & $B$ & $1.65 \pm 0.09$ & $16.01 \pm 0.06$ & FTN \\
\hline 4110 & 30 & $B$ & $1.95 \pm 0.31$ & $15.83 \pm 0.17$ & FTN \\
\hline 4525 & 60 & $B$ & $1.45 \pm 0.17$ & $16.15 \pm 0.13$ & FTN \\
\hline 5336 & 120 & $B$ & $1.09 \pm 0.11$ & $16.46 \pm 0.11$ & FTN \\
\hline 6389 & 180 & $B$ & $0.89 \pm 0.12$ & $16.68 \pm 0.15$ & FTN \\
\hline 7564 & 120 & $B$ & $0.68 \pm 0.16$ & $16.98 \pm 0.25$ & FTN \\
\hline 9429 & 220 & $B$ & $0.47 \pm 0.12$ & $17.38 \pm 0.28$ & FTN \\
\hline $1.11 \mathrm{E}+04$ & 310 & $B$ & $0.46 \pm 0.09$ & $17.41 \pm 0.22$ & FTN \\
\hline 507.8 & 10 & $V$ & $5.53 \pm 0.51$ & $14.55 \pm 0.1$ & FTN \\
\hline 8721 & 10 & V & $1.90 \pm 0.37$ & $15.71 \pm 0.21$ & FTN \\
\hline 3338 & 300 & $V$ & $2.30 \pm 0.24$ & $15.5 \pm 0.112$ & SRO \\
\hline 3654 & 300 & V & $1.86 \pm 0.21$ & $15.73 \pm 0.121$ & SRO \\
\hline 3964 & 300 & $V$ & $1.83 \pm 0.22$ & $15.75 \pm 0.133$ & SRO \\
\hline 4273 & 300 & $V$ & $1.81 \pm 0.21$ & $15.76 \pm 0.126$ & SRO \\
\hline 4582 & 300 & $V$ & $1.97 \pm 0.16$ & $15.67 \pm 0.091$ & SRO \\
\hline 4891 & 300 & V & $1.79 \pm 0.25$ & $15.77 \pm 0.151$ & SRO \\
\hline 5510 & 300 & $V$ & $2.10 \pm 0.21$ & $15.6 \pm 0.107$ & SRO \\
\hline 5819 & 300 & V & $1.87 \pm 0.21$ & $15.72 \pm 0.123$ & SRO \\
\hline 6128 & 300 & $V$ & $1.79 \pm 0.27$ & $15.77 \pm 0.163$ & SRO \\
\hline 230 & 60 & $V$ & $0.98 \pm 0.42$ & $16.43 \pm 0.45$ & KAIT \\
\hline 425 & 20 & $V$ & $8.14 \pm 1.13$ & $14.13 \pm 0.15$ & KAIT \\
\hline 522 & 20 & $V$ & $3.65 \pm 0.99$ & $15 \pm 0.29$ & KAIT \\
\hline 665.5 & 40 & $V$ & $2.65 \pm 0.44$ & $15.35 \pm 0.18$ & KAIT \\
\hline 910.6 & 60 & V & $3.33 \pm 0.43$ & $15.1 \pm 0.14$ & KAIT \\
\hline 201.2 & 10 & $R$ & $1.81 \pm 0.13$ & $15.58 \pm 0.08$ & FTN \\
\hline 231.8 & 10 & $R$ & $2.63 \pm 0.14$ & $15.17 \pm 0.06$ & FTN \\
\hline 264.8 & 10 & $R$ & $3.20 \pm 0.14$ & $14.96 \pm 0.05$ & FTN \\
\hline 844.8 & 30 & $R$ & $4.29 \pm 0.28$ & $14.64 \pm 0.07$ & FTN \\
\hline 1438 & 60 & $R$ & $2.52 \pm 0.19$ & $15.22 \pm 0.08$ & FTN \\
\hline 2339 & 120 & $R$ & $3.47 \pm 0.19$ & $14.87 \pm 0.06$ & FTN \\
\hline 3511 & 180 & $R$ & $2.73 \pm 0.176$ & $15.13 \pm 0.07$ & FTN \\
\hline 4204 & 30 & $R$ & $2.47 \pm 0.20$ & $15.24 \pm 0.09$ & FTN \\
\hline 4650 & 60 & $R$ & $2.17 \pm 0.18$ & $15.38 \pm 0.09$ & FTN \\
\hline 5521 & 120 & $R$ & $2.27 \pm 0.17$ & $15.33 \pm 0.08$ & FTN \\
\hline 6631 & 180 & $R$ & $1.84 \pm 0.15$ & $15.56 \pm 0.09$ & FTN \\
\hline 7564 & 120 & $R$ & $1.42 \pm 0.13$ & $15.84 \pm 0.1$ & FTN \\
\hline 8463 & 30 & $R$ & $1.33 \pm 0.14$ & $15.91 \pm 0.11$ & FTN \\
\hline 9015 & 30 & $R$ & $1.02 \pm 0.13$ & $16.2 \pm 0.14$ & FTN \\
\hline 9378 & 60 & $R$ & $0.98 \pm 0.14$ & $16.24 \pm 0.15$ & FTN \\
\hline 9962 & 120 & $R$ & $1.05 \pm 0.097$ & $16.17 \pm 0.1$ & FTN \\
\hline $1.09 \mathrm{E}+04$ & 180 & $R$ & $0.93 \pm 0.086$ & $16.3 \pm 0.1$ & FTN \\
\hline $1.19 \mathrm{E}+04$ & 120 & $R$ & $0.68 \pm 0.069$ & $16.64 \pm 0.11$ & FTN \\
\hline $1.24 \mathrm{E}+04$ & 30 & $R$ & $0.80 \pm 0.11$ & $16.46 \pm 0.15$ & FTN \\
\hline $4.26 \mathrm{E}+04$ & 1800 & $R$ & $0.11 \pm 0.0099$ & $18.65 \pm 0.1$ & LT \\
\hline $5.46 \mathrm{E}+04$ & 3600 & $R$ & $0.073 \pm 0.0068$ & $19.06 \pm 0.1$ & LT \\
\hline $6.43 E+04$ & 1800 & $R$ & $0.041 \pm 0.0077$ & $19.68 \pm 0.2$ & LT \\
\hline 642.9 & 180 & $R$ & $5.94 \pm 0.17$ & $14.29 \pm 0.031$ & SRO \\
\hline 839.1 & 180 & $R$ & $4.38 \pm 0.15$ & $14.62 \pm 0.038$ & SRO \\
\hline 1028 & 180 & $R$ & $3.21 \pm 0.16$ & $14.95 \pm 0.053$ & SRO \\
\hline 1219 & 180 & $R$ & $2.73 \pm 0.12$ & $15.13 \pm 0.048$ & SRO \\
\hline 1408 & 180 & $R$ & $2.43 \pm 0.13$ & $15.26 \pm 0.058$ & SRO \\
\hline 1723 & 300 & $R$ & $2.76 \pm 0.12$ & $15.12 \pm 0.047$ & SRO \\
\hline 2039 & 300 & $R$ & $3.51 \pm 0.15$ & $14.86 \pm 0.047$ & SRO \\
\hline 2348 & 300 & $R$ & $3.62 \pm 0.11$ & $14.82 \pm 0.034$ & SRO \\
\hline 2657 & 300 & $R$ & $3.89 \pm 0.14$ & $14.75 \pm 0.038$ & SRO \\
\hline 2966 & 300 & $R$ & $3.46 \pm 0.14$ & $14.87 \pm 0.045$ & SRO \\
\hline 99.42 & 20 & $R$ & $0.52 \pm 0.18$ & $16.93 \pm 0.36$ & KAIT \\
\hline 200.8 & 20 & $R$ & $2.04 \pm 0.13$ & $15.45 \pm 0.07$ & KAIT \\
\hline 307 & 20 & $R$ & $4.17 \pm 0.15$ & $14.67 \pm 0.04$ & KAIT \\
\hline 404 & 20 & $R$ & $6.86 \pm 0.44$ & $14.13 \pm 0.07$ & KAIT \\
\hline 499 & 20 & $R$ & $7.12 \pm 0.26$ & $14.09 \pm 0.04$ & KAIT \\
\hline 594 & 20 & $R$ & $5.66 \pm 0.16$ & $14.34 \pm 0.03$ & KAIT \\
\hline 691 & 20 & $R$ & $5.45 \pm 0.15$ & $14.38 \pm 0.03$ & KAIT \\
\hline 790 & 20 & $R$ & $4.49 \pm 0.17$ & $14.59 \pm 0.04$ & IT \\
\hline
\end{tabular}


Table 1

(Continued)

\begin{tabular}{|c|c|c|c|c|c|}
\hline $\begin{array}{l}\Delta t^{\mathrm{b}} \\
(\mathrm{s})\end{array}$ & $\begin{array}{c}\text { Exposure } \\
\text { (s) }\end{array}$ & Filter & $\begin{array}{l}\text { Flux }^{c} \\
(m J y)\end{array}$ & Magnitude $^{c}$ & Telescope \\
\hline 889 & 20 & $R$ & $3.81 \pm 0.14$ & $14.77 \pm 0.04$ & KAIT \\
\hline 998 & 20 & $R$ & $3.57 \pm 0.16$ & $14.84 \pm 0.05$ & KAIT \\
\hline 106 & 50 & $R$ & $0.516 \pm 0.27$ & $16.94 \pm 0.55$ & S-LOTIS \\
\hline 167 & 40 & $R$ & $1.34 \pm 0.26$ & $15.9 \pm 0.21$ & S-LOTIS \\
\hline 207.3 & 20 & $R$ & $2.13 \pm 0.20$ & $15.4 \pm 0.1$ & S-LOTIS \\
\hline 234 & 20 & $R$ & $2.61 \pm 0.26$ & $15.18 \pm 0.11$ & S-LOTIS \\
\hline 261.3 & 20 & $R$ & $3.45 \pm 0.19$ & $14.87 \pm 0.06$ & S-LOTIS \\
\hline 308.5 & 60 & $R$ & $4.17 \pm 0.23$ & $14.67 \pm 0.06$ & S-LOTIS \\
\hline 375.3 & 60 & $R$ & $5.56 \pm 0.15$ & $14.36 \pm 0.03$ & S-LOTIS \\
\hline 442 & 60 & $R$ & $7.06 \pm 0.13$ & $14.1 \pm 0.02$ & S-LOTIS \\
\hline 509.2 & 60 & $R$ & $6.93 \pm 0.19$ & $14.12 \pm 0.03$ & S-LOTIS \\
\hline 575.9 & 60 & $R$ & $5.76 \pm 0.16$ & $14.32 \pm 0.03$ & S-LOTIS \\
\hline 643.1 & 60 & $R$ & $5.61 \pm 0.15$ & $14.35 \pm 0.03$ & S-LOTIS \\
\hline 709.9 & 60 & $R$ & $4.97 \pm 0.14$ & $14.48 \pm 0.03$ & S-LOTIS \\
\hline 777.1 & 60 & $R$ & $4.41 \pm 0.16$ & $14.61 \pm 0.04$ & S-LOTIS \\
\hline 844 & 60 & $R$ & $4.06 \pm 0.15$ & $14.7 \pm 0.04$ & S-LOTIS \\
\hline 911.2 & 60 & $R$ & $3.54 \pm 0.16$ & $14.85 \pm 0.05$ & S-LOTIS \\
\hline 977.8 & 60 & $R$ & $3.26 \pm 0.15$ & $14.94 \pm 0.05$ & S-LOTIS \\
\hline 1078 & 120 & $R$ & $2.71 \pm 0.075$ & $15.14 \pm 0.03$ & S-LOTIS \\
\hline 1245 & 180 & $R$ & $2.52 \pm 0.19$ & $15.22 \pm 0.08$ & S-LOTIS \\
\hline 1412 & 120 & $R$ & $2.38 \pm 0.11$ & $15.28 \pm 0.05$ & S-LOTIS \\
\hline 1516 & 120 & $R$ & $2.49 \pm 0.14$ & $15.23 \pm 0.06$ & S-LOTIS \\
\hline 1713 & 180 & $R$ & $2.54 \pm 0.12$ & $15.21 \pm 0.05$ & S-LOTIS \\
\hline 1880 & 120 & $R$ & $3.14 \pm 0.14$ & $14.98 \pm 0.05$ & S-LOTIS \\
\hline 2014 & 120 & $R$ & $3.41 \pm 0.16$ & $14.89 \pm 0.05$ & S-LOTIS \\
\hline 2147 & 120 & $R$ & $3.26 \pm 0.15$ & $14.94 \pm 0.05$ & S-LOTIS \\
\hline 2248 & 60 & $R$ & $3.50 \pm 0.26$ & $14.86 \pm 0.08$ & S-LOTIS \\
\hline $2.46 \mathrm{E}+05$ & 900 & $R$ & $0.0069 \pm 0.00078$ & $21.62 \pm 0.12$ & Gemini \\
\hline 594.2 & 10 & $I$ & $7.10 \pm 0.33$ & $13.89 \pm 0.05$ & FTN \\
\hline 1014 & 30 & $I$ & $4.24 \pm 0.13$ & $14.45 \pm 0.03$ & FTN \\
\hline 1632 & 60 & $I$ & $3.53 \pm 0.16$ & $14.65 \pm 0.05$ & FTN \\
\hline 2749 & 120 & $I$ & $4.78 \pm 0.13$ & $14.32 \pm 0.03$ & FTN \\
\hline 4307 & 30 & $I$ & $2.99 \pm 0.14$ & $14.83 \pm 0.05$ & FTN \\
\hline 4854 & 60 & $I$ & $2.83 \pm 0.10$ & $14.89 \pm 0.04$ & FTN \\
\hline 5776 & 120 & $I$ & $2.85 \pm 0.079$ & $14.88 \pm 0.03$ & FTN \\
\hline 6977 & 180 & $I$ & $2.11 \pm 0.097$ & $15.21 \pm 0.05$ & FTN \\
\hline 8802 & 10 & $I$ & $1.50 \pm 0.14$ & $15.58 \pm 0.1$ & FTN \\
\hline 9124 & 30 & $I$ & $1.58 \pm 0.073$ & $15.52 \pm 0.05$ & FTN \\
\hline 9530 & 60 & $I$ & $1.23 \pm 0.068$ & $15.79 \pm 0.06$ & FTN \\
\hline $1.02 \mathrm{E}+04$ & 120 & $I$ & $1.11 \pm 0.071$ & $15.91 \pm 0.07$ & FTN \\
\hline $1.12 \mathrm{E}+04$ & 180 & $I$ & $0.98 \pm 0.045$ & $16.04 \pm 0.05$ & FTN \\
\hline $4.82 \mathrm{E}+04$ & 3600 & $I$ & $0.13 \pm 0.0099$ & $18.2 \pm 0.08$ & LT \\
\hline $5.89 \mathrm{E}+04$ & 1800 & $I$ & $0.076 \pm 0.0070$ & $18.82 \pm 0.1$ & LT \\
\hline 6877 & 180 & $I$ & $2.53 \pm 0.17$ & $15.01 \pm 0.074$ & SRO \\
\hline 174 & 20 & $I$ & $2.01 \pm 0.28$ & $15.26 \pm 0.15$ & KAIT \\
\hline 274 & 20 & $I$ & $4.56 \pm 0.29$ & $14.37 \pm 0.07$ & KAIT \\
\hline 371 & 20 & $I$ & $7.72 \pm 0.50$ & $13.8 \pm 0.07$ & KAIT \\
\hline 468 & 20 & $I$ & $9.80 \pm 0.45$ & $13.54 \pm 0.05$ & KAIT \\
\hline 563 & 20 & $I$ & $7.51 \pm 0.35$ & $13.83 \pm 0.05$ & KAIT \\
\hline 658 & 20 & $I$ & $7.17 \pm 0.26$ & $13.88 \pm 0.04$ & KAIT \\
\hline 757 & 20 & $I$ & $5.96 \pm 0.33$ & $14.08 \pm 0.06$ & KAIT \\
\hline 856 & 20 & $I$ & $5.14 \pm 0.28$ & $14.24 \pm 0.06$ & KAIT \\
\hline 955 & 20 & $I$ & $4.52 \pm 0.33$ & $14.38 \pm 0.08$ & KAIT \\
\hline $2.46 \mathrm{E}+05$ & 900 & $I$ & $0.012 \pm 0.0011$ & $20.8 \pm 0.1$ & Gemini \\
\hline $1.97 \mathrm{E}+06$ & 1800 & $I$ & $>0.00086$ & $>23.68$ & Gemini \\
\hline
\end{tabular}

Notes.

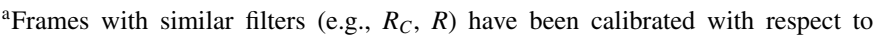
a common set of standard stars in the reference filter listed. Data were taken with the Faulkes Telescope North (FTN), the Sonoita Research Observatory Telescope (SRO), the Katzman Automatic Imaging Telescope (KAIT), Super-LOTIS (S-LOTIS), the Liverpool Telescope (LT), and Gemini. See Section 2.2 for further details.

${ }^{\mathrm{b}} \Delta t$ is the midpoint of the exposure in time elapsed (s) since the Swift-BAT trigger. ${ }^{\mathrm{c}}$ Magnitudes and fluxes have been corrected for Galactic absorption using $E(B-$ $V)=0.98 \mathrm{mag}$, which corresponds to $A_{B}=4.24, A_{V}=3.17, A_{R}=2.58$, and $A_{I}=1.92 \mathrm{mag}$.
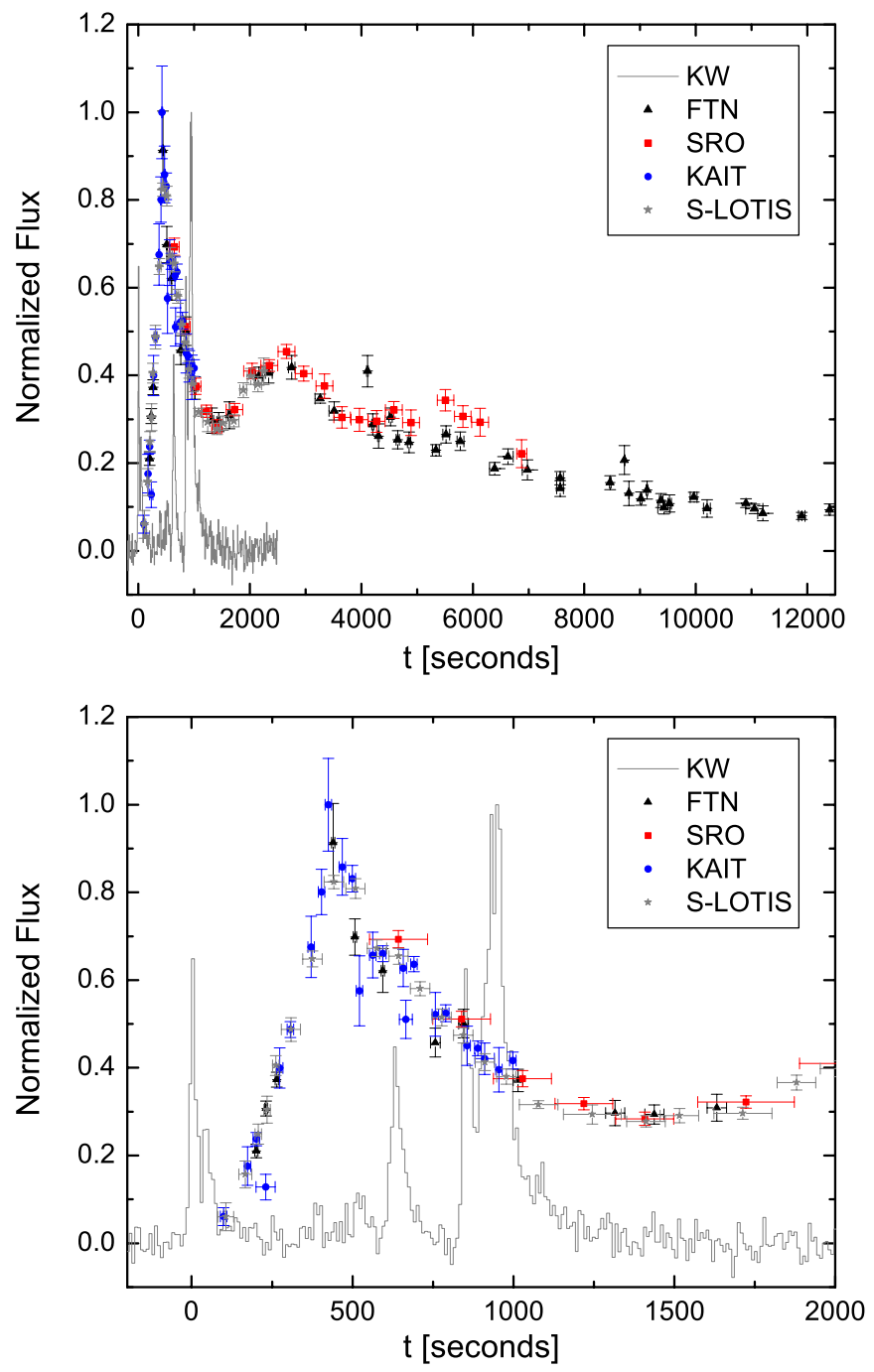

Figure 4. Normalized optical (symbols) and KW $\gamma$-ray light curves (18-1160 keV; gray line) plotted on a linear scale for temporal comparison. Colors indicate the telescope used. Late-time LT and Gemini data points at $T_{0}+10^{4}-10^{6} \mathrm{~s}$ are omitted for clarity.

(A color version of this figure is available in the online journal.)

to the corresponding filter and unfiltered frames have been calibrated with respect to the $R$ band ( $\mathrm{Li}$ et al. 2003).

\subsubsection{Faulkes Telescope North}

The $2 \mathrm{~m}$ Faulkes Telescope North (FTN) began monitoring at $T_{0}+196 \mathrm{~s}$, automatically identifying the optical afterglow (Mundell et al. 2009; Cano et al. 2009), continuing observations until $T_{0}+1.2 \times 10^{4} \mathrm{~s}$. A series of images in alternating $B V R I$ filters were taken, with exposure times in the range of 10-180 s.

\subsubsection{SRO/AAVSO}

The $0.35 \mathrm{~m}$ SRO telescope began observing at $T_{0}+540 \mathrm{~s}$ lasting for about an hour (Henden et al. 2009). They observed a series of $V, R_{C}$, and $I_{C}$ images with exposure times of 180 and $300 \mathrm{~s}$. Images have been calibrated with respect to the $V, R$, and $I$ filters.

\subsubsection{Liverpool Telescope}

The $2 \mathrm{~m}$ Liverpool Telescope (LT) provided late-time coverage from $T_{0}+(4.2-6.4) \times 10^{4} \mathrm{~s}$. Five images were taken with 
Table 2

Optical Light Curve Multi-component-fit Parameters ${ }^{\mathrm{a}}$

\begin{tabular}{lcccrr}
\hline \hline & $\alpha_{\text {rise }}$ & $\alpha_{\text {decay }}$ & $\begin{array}{c}t_{\text {break }}^{\mathrm{b}} \\
(\mathrm{s})\end{array}$ & \multicolumn{1}{c}{$\begin{array}{c}t_{\text {peak }}^{\mathrm{c}} \\
(\mathrm{s})\end{array}$} & Normalization \\
\hline Component 1 & $-2.37 \pm 0.13$ & $1.83 \pm 0.14$ & $450 \pm 19$ & $480 \pm 19$ & $13 \pm 0.19$ \\
Component 2 & $-4.17 \pm 0.92$ & $1.57 \pm 0.57$ & $2200 \pm 220$ & $2600 \pm 220$ & $5.7 \pm 0.84$ \\
Component 3 & $-15.29 \pm 12.7$ & $1.48 \pm 0.81$ & $5080 \pm 380$ & $5800 \pm 380$ & $1.0 \pm 0.54$ \\
\hline
\end{tabular}

Notes.

a The reduced $\chi^{2}$ for the fit is 1.43 for 75 dof and is performed on the $R$ and $I$ photometry. See Figure 5 and Section 2.3 for details.

${ }^{\mathrm{b}}$ Break time of the Beuermann function fit of each component.

c Time of the light curve peak.

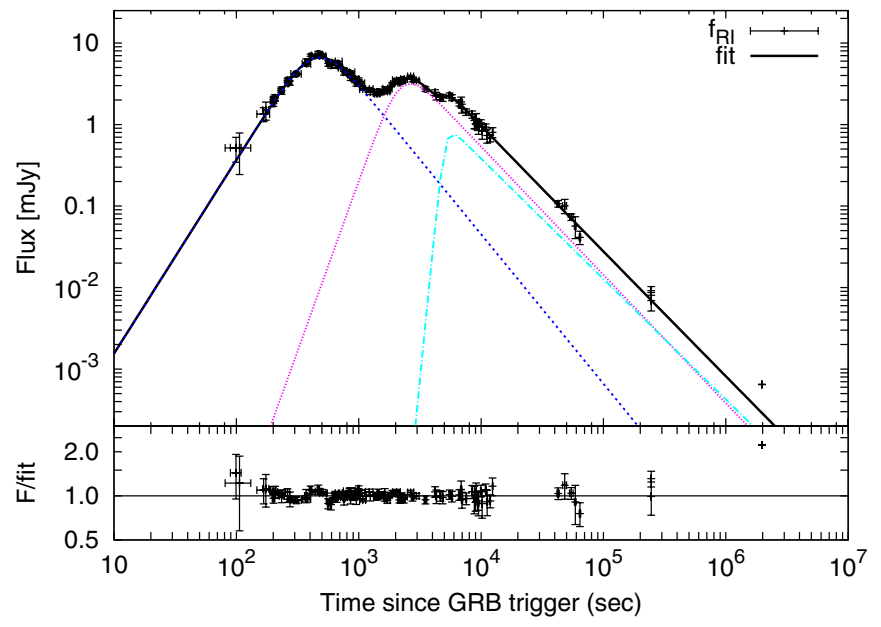

Figure 5. Multi-component fit to the $R$ and $I$ (shifted) optical light curve of GRB 091024A. Individual components are in color (dashed) and the total in black (solid). The last data point is an upper limit and is not used to constrain the model. Observed photometric data are summarized in Table 1 and model fits are reported in Table 2.

(A color version of this figure is available in the online journal.)

exposures of 1800 or $3600 \mathrm{~s}$, detecting the afterglow in both the $R$ and $I$ bands.

\subsubsection{Gemini Imaging}

The $8 \mathrm{~m}$ Gemini North telescope provided additional latetime coverage with a detection in $r^{\prime}$ and $i^{\prime}$ from $5 \times 180 \mathrm{~s}$ exposures beginning at $T_{0}+2.8$ days and an upper limit in $i^{\prime}$ from a $9 \times 200 \mathrm{~s}$ exposure beginning at $T_{0}+22.8$ days. Data was reduced using the standard Gemini pipeline tools within the IRAF/gemini package.

\subsection{Optical Light Curve Fitting}

We model the optical light curve with superimposed broken power law components. In order to better characterize the optical behavior, we used the best-sampled optical bands ( $R$ and $I$ ). No strong evidence for color change is observed in these bands during the peak episodes in the $10^{2}-10^{4} \mathrm{~s}$ time interval. For that reason we rigidly shift the $I$-band flux to the $R$-band flux (by a factor 0.75 ) before performing the multi-component fit of the light curve, in order to have the best sampling of the different peaks. The results of the fit are reported in Table 2 and overlaid with the optical observations in Figure 5. As can be seen, the behavior in the optical band is better described by the sum of three broken power laws $\left(\chi_{\text {reduced }}^{2}=1.43\right.$, dof $\left.=75\right)$. The final data point obtained by Gemini $\left(T_{0}+2 \times 10^{6} \mathrm{~s}\right)$ is an upper limit and not used in the model fitting.
If we consider all the optical bands individually, we note that evidence of some color change at early times becomes greater. While variation between the $R$ and $I$ bands is compatible with the uncertainties of the combined fit (Table 2), there are small variations in the rising slopes $(\alpha)$ of individual filters for $t<400 \mathrm{~s}$ between bluer $(B$ and $V$ ) and redder ( $R$ and $I$ ) filters: $\alpha_{V} \approx-3.3, \alpha_{R}=-2.35 \pm 0.15$, and $\alpha_{I}=-2.53 \pm 0.53$. After the first peak we do not have good sampling of bluer filters, and it becomes difficult to compare their light curve shapes with the parameters of the composite fitted function reported in Table 2 for the redder filters. This might indicate some sort of color evolution before and around the time of first peak that is visible only at shorter wavelengths. At late times no color evolution is detected. Observations with simultaneous color information, such as the RINGO3 polarimeter (Arnold et al. 2012), would greatly improve the availability of color information.

\subsection{Spectroscopy and Redshift Determination}

\subsubsection{Keck Spectroscopy}

We obtained a spectrum of GRB 091024A with the LowResolution Imaging Spectrometer (LRIS; Oke et al. 1995) mounted on the $10 \mathrm{~m}$ Keck I telescope beginning at 11:01 on 2009 October $24\left(T_{0}+2.08 \mathrm{hr}\right)$. We employed the $5600 \AA$ dichroic beam splitter, with the 400 lines $\mathrm{mm}^{-1}$ grism blazed at $3400 \AA$ on the blue side and the 400 lines $\mathrm{mm}^{-1}$ grating blazed at $8500 \AA$ on the red side (corresponding to $\sim 7 \AA$ resolution on both ends). A total of $1200 \mathrm{~s}(1250 \mathrm{~s})$ exposure time was accumulated in multiple images for the blue (red) sides, covering a combined wavelength range from the atmospheric cutoff to $\sim 10000 \AA$.

All spectra were reduced in the $\operatorname{IRAF}^{21}$ environment using standard routines. Cosmic rays were removed using the LA Cosmic routine (van Dokkum 2001). Spectra were extracted optimally (Horne 1986), and wavelength calibration was performed first relative to $\mathrm{Hg}-\mathrm{Cd}-\mathrm{Zn}-\mathrm{Ar}$ lamps and then adjusted slightly based on night-sky lines in each individual image. Both air-to-vacuum and heliocentric corrections were then applied to all spectra. Flux calibration was performed by comparison with the spectrum of a spectrophotometric standard star. Finally, telluric atmospheric absorption features were removed through division by the standard-star spectrum in the relevant regions (Wade \& Horne 1988; Matheson et al. 2000).

We identify a series of strong atomic absorption transitions which are presented in Table 3 (see Figure 6). All of the detected components are consistent with a redshift $z=1.0924 \pm 0.0002$.

\footnotetext{
21 IRAF is distributed by the National Optical Astronomy Observatory, which is operated by the Association for Research in Astronomy, Inc., under cooperative agreement with the National Science Foundation (NSF).
} 
Table 3

Keck/LRIS Absorption-line Identifications

\begin{tabular}{cccc}
\hline $\begin{array}{c}\text { Observed Wavelength } \\
(\AA)\end{array}$ & $\begin{array}{c}\text { Identification } \\
(\AA)\end{array}$ & $\begin{array}{c}\text { Vacuum Wavelength } \\
(\AA)\end{array}$ & $\begin{array}{c}\text { Rest-frame Equivalent Width } \\
(\AA)\end{array}$ \\
\hline $4906.08 \pm 0.40$ & Fe II & 2344.704 & $1.43 \pm 0.17$ \\
$4968.85 \pm 0.55$ & Fe II & 2374.461 & $1.02 \pm 0.32$ \\
$4985.74 \pm 0.40$ & Fe II & 2382.765 & $2.11 \pm 0.11$ \\
$5412.51 \pm 0.40$ & Fe II & 2586.650 & $1.67 \pm 0.05$ \\
$5440.37 \pm 0.40$ & Fe II & 2600.173 & $2.05 \pm 0.12$ \\
$5851.19 \pm 0.49$ & Mg II & 2796.352 & $3.02 \pm 0.25$ \\
$5866.18 \pm 0.42$ & Mg II & 2803.531 & $2.32 \pm 0.35$ \\
$5968.43 \pm 0.30$ & Mg I & 2852.964 & $1.32 \pm 0.18$ \\
$8232.54 \pm 0.20$ & Ca II & 3934.777 & $1.73 \pm 0.03$ \\
$8305.99 \pm 0.20$ & Ca II & 3969.591 & $1.17 \pm 0.03$ \\
\hline
\end{tabular}

Note. ${ }^{a}$ Morton (1991)

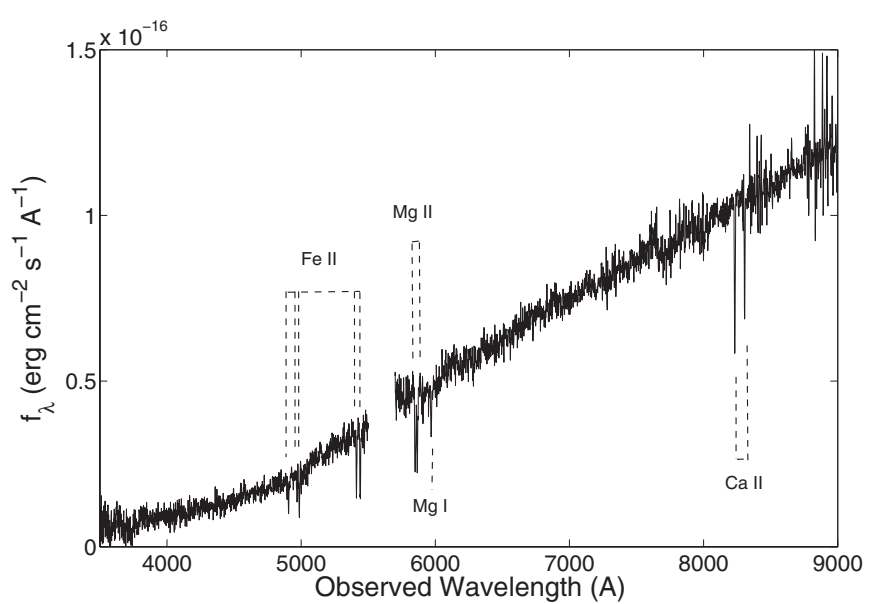

Figure 6. Spectrum of GRB 091024A obtained with LRIS on the $10 \mathrm{~m}$ Keck I telescope at $T_{0}+2.08 \mathrm{hr}$. Strong absorption features and components (Table 3) imply $z=1.0924 \pm 0.0002$.

The flux below $3500 \AA$ is consistent with zero, and the lack of Ly- $\alpha$ absorption redward of this value limits the host-galaxy redshift of GRB 091024A to $z \lesssim 1.9$.

\subsubsection{Gemini Spectroscopy}

Gemini-N equipped with the GMOS camera began to perform spectroscopic observations at $T_{0}+2.38 \mathrm{hr}$. The target was visible in the $60 \mathrm{~s} i$-band acquisition image and placed in a $1^{\prime \prime}$ slit. We used the $R 400 / 800$ grating configuration which allowed us to cover the 6000-10000 $\AA$ wavelength range with a resolution of $R \approx 1200$ at the midpoint. Two $900 \mathrm{~s}$ spectra were obtained, followed by a flatfield and a comparison lamp spectrum with the same configuration. All of the raw data were processed with the dedicated GEMINI and GMOS tools inside the IRAF environment. Flatfielding, sky-background subtraction, and cosmic-ray rejection were performed, and one-dimensional spectra of the afterglow and the comparison lamp were extracted using the APALL task. We derived the wavelength solution and applied it to our afterglow spectra.

The resulting spectra were co-added to increase the $S / N$. In the entire wavelength range spanned by our data, we identified $\mathrm{Mg} \mathrm{I}$ and $\mathrm{Ca} \mathrm{H}$ and $\mathrm{K}$ absorption features. We fit Voigt profiles to these features, resulting in the following rest-frame equivalent widths: $W_{\mathrm{MgI} \lambda 2853}=1.17 \pm 0.62, W_{\text {CaII } \lambda 3934}=1.38 \pm 0.44$, $W_{\text {CaII } \lambda 3969}=1.66 \pm 0.82$. These strong lines were consistent with a common redshift for the host galaxy of $z=1.092$.

\section{DATA ANALYSIS}

\subsection{Temporal Analysis}

The most obvious characteristic that sets GRB 091024A apart from other bursts is its extremely long duration, with an episode of $\gamma$-ray emission coincident with the Swift trigger as well as two subsequent emission episodes peaking at about $T_{0}+650$ and $950 \mathrm{~s}$ in both Fermi/GBM and KW. From the GBM data alone it is difficult to tell whether there is low-level emission during the periods between the emission episodes due to the large fluctuations in the background. In contrast, the KW light curves have a very flat baseline and, as detailed below, we find significant low-level activity in the long interpulse interval (see Figure 1). In addition, we find that the duration of the second emission episode is underestimated in the GBM data, and we perform all of our analyses with the KW derived durations.

In order to probe the activity of the central engine, we perform a power-spectrum analysis of the unmasked Swift-BAT and KW $\gamma$-ray data in the time domain (Li 2001). Specifically, we calculate the fractional power density of the signal for the entire time interval, as well as various temporal epochs and spectral regimes. This quantity gives a measure of the intrinsic time variability in the signal (see Margutti et al. 2008 and Margutti $2009^{22}$ for further details) from which we can infer the activity of the central engine powering the GRB. Using this method, we find two short and two long characteristic timescales at $0.6 \pm 0.2$ and $1.4 \pm 0.2 \mathrm{~s}$, and $7 \pm 0.03$ and $20 \pm 0.03 \mathrm{~s}$, respectively (see Figure 7). Errors are estimated using Monte Carlo simulations.

Perhaps more importantly, we find different variability timescales at different temporal epochs during the burst emission. The first $\left(T_{0}-200\right.$ to $\left.T_{0}+250 \mathrm{~s}\right)$ and third $\left(T_{0}+800\right.$ to $T_{0}+1200 \mathrm{~s}$ ) slices include the first and final emission episode, while the second $\left(T_{0}+250\right.$ to $\left.T_{0}+800 \mathrm{~s}\right)$ includes the low-level emission period and the weak second emission episode. The epochs with large amounts of emission show variability at very short timescales, while the second epoch shows only longer variability timescales, behaving in a similar way as the empirical luminosity-variability relation (Fenimore \& Ramirez-Ruiz

\footnotetext{
$22 \mathrm{PhD}$ thesis. Available at http://boa.unimib.it/handle/10281/7465. For details on this method, see specifically Chapter 6. Most inferences based on standard tools like the Fast Fourier Transform (FFT) require stationary signals where the duration is much longer than the typical pulse width, which is not the case for GRBs. Additionally, care must be used in the interpretation of the Fourier spectrum of an aperiodic signal in the time domain, since GRBs are strictly non-periodic (see, e.g., Li \& Muraki 2001). The Time in the Time Domain (TTD) analysis is designed to overcome these limitations and is optimized to study the variability timescale of short, non-repetitive, non-stationary signals like GRBs.
} 
Table 4

Konus-Wind Time-integrated Spectral Parameters for Each $\gamma$-Ray Emission Episode ${ }^{\mathrm{a}}$

\begin{tabular}{|c|c|c|c|c|c|c|c|}
\hline $\begin{array}{l}\text { GBM Time Interval } \\
\text { (s) }\end{array}$ & $\begin{array}{c}\text { KW Time Interval } \\
\text { (s) }\end{array}$ & $\begin{array}{l}\Delta t \\
(\mathrm{~s})\end{array}$ & $\alpha_{\mathrm{CPL}}$ & $\begin{array}{c}E_{\text {peak,CPL }} \\
(\mathrm{keV})\end{array}$ & $\alpha_{\mathrm{GRBM}}$ & $\begin{array}{c}E_{\text {peak, GRBM }} \\
(\mathrm{keV})\end{array}$ & $\beta_{\mathrm{GRBM}}$ \\
\hline$(-3.8,67.8)$ & $(-6.6,65.0)$ & 71.6 & $-1.07_{-0.08}^{+0.09}$ & $495_{-69}^{+106}$ & $-1.06_{-0.08}^{+0.10}$ & $474_{-74}^{+111}$ & -2.5 \\
\hline$(622.7,664.7)$ & $(619.9,661.9)$ & 42 & $-1.59_{-0.11}^{+0.10}$ & $374_{-128}^{+490}$ & $-1.58_{-0.10}^{+0.13}$ & $495_{-140}^{+494}$ & -2.5 \\
\hline$(838.8,1070.2)$ & $(836.0,1067.4)$ & 231.4 & $-1.42_{-0.4}^{+0.4}$ & $246_{-26}^{+26}$ & $-1.38_{-0.05}^{+0.06}$ & $216_{-25}^{+31}$ & -2.5 \\
\hline$(-6.1,82.2)$ & $(-8.9,79.4)$ & 88.3 & $-1.10_{-0.08}^{+0.09}$ & $527_{-79}^{+125}$ & $-1.09_{-0.08}^{+0.10}$ & $508_{-84}^{+130}$ & -2.5 \\
\hline$(612.1,718.1)$ & $(609.4,715.3)$ & 105.9 & $-1.61_{-0.13}^{+0.17}$ & $184_{-48}^{+137}$ & $-1.57_{-0.17}^{+1.96}$ & $161_{-87}^{+148}$ & -2.5 \\
\hline$(835.1,1312.8)$ & $(833.1,1310.0)$ & 476.9 & $-1.49_{-0.05}^{+0.04}$ & $255_{-28}^{+41}$ & $-1.46_{-0.06}^{+0.06}$ & $230_{-34}^{+46}$ & -2.5 \\
\hline$(82.2,606.6)$ & $(79.4,609.4)$ & 524.4 & $-1.73_{-0.12}^{+0.13}$ & $\ldots$ & $\ldots$ & $\ldots$ & $\ldots$ \\
\hline
\end{tabular}

Notes.

${ }^{a}$ The first two sets of parameters are fits over the approximate Fermi/GBM durations and the latter sets are fits using the Bayesian Block derived durations. Each time interval is fit with a cutoff power law (CPL) and GRBM model with $\beta_{\mathrm{GRBM}}=-2.5$. The last line is a simple power law fit over the interval between the first and second emission episodes. Errors are approximated with the bootstrap method and given to the $1 \sigma$ confidence level.

b Seconds with respect to the first Fermi-GBM trigger of GRB 091024A.

${ }^{c}$ Seconds with respect to the Konus-Wind trigger.

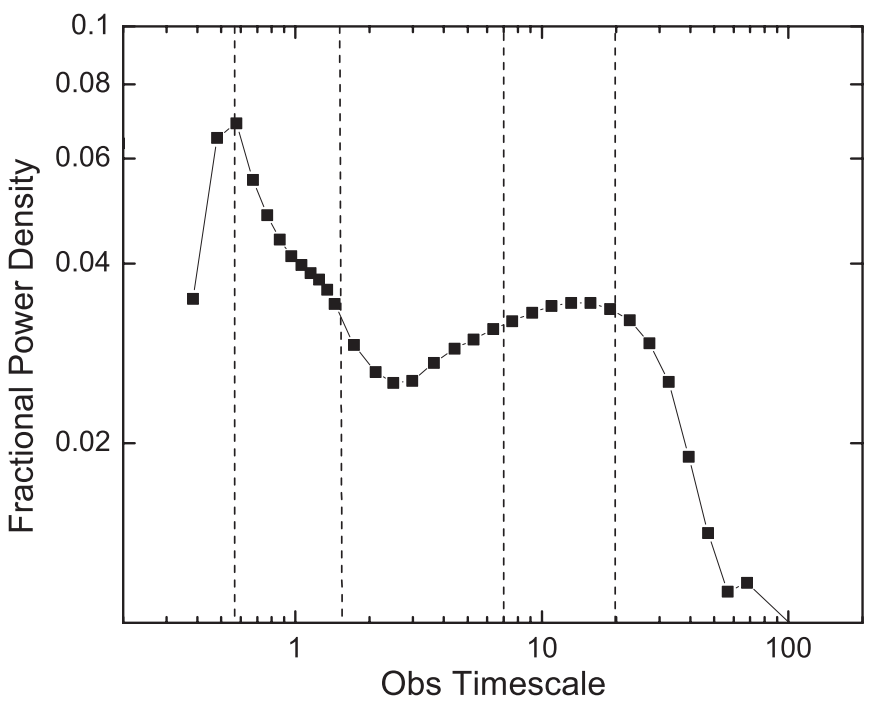

Figure 7. Summary of the fractional power density analysis on the entire Swift/ BAT non-mask weighted data from $-200<T_{0}<1200 \mathrm{~s}$. The four timescales of $0.6 \pm 0.2,1.4 \pm 0.2,7 \pm 0.03$, and $20 \pm 0.03 \mathrm{~s}$ are indicated with dashed lines. Errors are estimated by Monte Carlo simulations.

2000; Reichart et al. 2001; Guidorzi et al. 2006a). The strong similarity in short variability timescales for the first and third episodes suggests that these episodes share a common origin and that the central engine powering the beginning of the burst likely reactivated to produce the later emission. We also performed this analysis over the individual BAT and KW energy channels. We find consistency with the trends in the full signal and additionally note that the softest energy channels $(15-25 \mathrm{keV}$ in BAT, $18-70 \mathrm{keV}$ in $\mathrm{KW}$ ) show little to no fractional power at low timescales, indicating less intrinsic variability at these energies.

\subsection{Spectral Analysis}

\subsubsection{Pulse Properties}

We performed both time-integrated and time-resolved analyses on the KW and BAT spectra. We do not include a joint analysis with Fermi-GBM spectra due to the rapidly changing background caused by the motion of the spacecraft, as discussed above. First, we consider the spectral parameters of the three emission episodes of the KW data. The spectra were modeled with cutoff power law (CPL) and Band (with $\beta=-2.5$ ) models using the three-channel data. For the Band model (Band et al. 1993) with fixed $\beta=-2.5$ we find $\alpha=\left(-1.09_{-0.08}^{+0.10},-1.57_{-0.17}^{+1.96},-1.46_{-0.06}^{+0.06}\right)$ and $E_{\text {peak }}=$ $\left(508_{-84}^{+130}, 161_{-87}^{+148}, 230_{-34}^{+46}\right)$ for the first, second, and third $\gamma$-ray episodes. The results show mild shallow-to-steep evolution in $\alpha$ and a softening of the second and third emission episodes. Both model types are consistent with each other and with the values reported by the GBM team (Gruber et al. 2011); full model parameters are summarized in Table 4. The derived KW fluence in the three emission episodes are $3.05_{-0.35}^{+0.24}, 1.30_{-0.14}^{+0.26}$, and $8.78_{-0.40}^{+0.52} \times 10^{-5} \mathrm{erg} \mathrm{cm}^{-2}$ in the $20 \mathrm{keV}$ to $10 \mathrm{MeV}$ band.

Second, we performed a more refined time-resolved analysis on the KW-BAT joint spectra, selecting smaller time bins using a Bayesian Block technique and combining bins as necessary to achieve sufficiently high $\mathrm{S} / \mathrm{N}$ to properly perform the statistical analysis. The error bars are larger in the time-resolved analysis and it is difficult to ascertain the true behavior of the spectrum, but the values of both $\alpha$ and $E_{\text {peak }}$ are consistent with the timeintegrated analysis of the entire first episode and with typical spectral parameters found in other bursts. Further evidence in support of the softening spectrum is found in the reduction of the hardness ratio between the different KW detectors. The results are summarized in Table 5.

\subsubsection{Interpulse Emission}

We also investigate the interpulse emission and find significant levels of faint emission between the first and second emission episodes $(\sim 7 \sigma$ in the $\mathrm{KW} \mathrm{G1}$ detector and $\sim 6 \sigma$ in the G2 detector). Since the level of emission is extremely low, we cannot perform a time resolved analysis of the entire interval and we take the entire interpulse region as one spectral bin. This emission is best fit by a simple power law with photon index of $-1.73_{-0.12}^{+0.13}$ and over its roughly $530 \mathrm{~s}$ has a fluence of $2.64_{-0.67}^{+1.10} \times 10^{-5} \mathrm{erg} \mathrm{cm}^{-2}$ in the $20 \mathrm{keV}$ to $10 \mathrm{MeV}$ band. This is a clear indication that the central engine may not cease all activity but may simply suffer from a temporary reduction in accretion rate until later times (López-Cámara et al. 2010), further supporting the conclusions from the fractional power density analysis of underlying timescales in the $\gamma$-ray emission. 
Table 5

Time-resolved Spectral Parameters for Joint Fits of First Emission Episode ${ }^{\mathrm{a}}$

\begin{tabular}{lccccc}
\hline \hline $\begin{array}{l}\text { Time Interval } \\
(\mathrm{s})\end{array}$ & Model & $\alpha$ & $\begin{array}{c}E_{\text {peak }} \\
(\mathrm{keV})\end{array}$ & $\chi^{2} /$ dof & Instrument \\
\hline$(-8.887,-2.999)$ & $\mathrm{CPL}$ & $-1.24_{-0.26}^{+0.39}$ & $572(>193)$ & $78.7 / 57$ & KW+BAT \\
$(-2.999,8.777)$ & $\mathrm{CPL}$ & $-0.93_{-0.11}^{+0.11}$ & $465_{-91}^{+140}$ & $55.5 / 57$ & KW+BAT \\
$(8.777,23.497)$ & $\mathrm{CPL}$ & $-1.04_{-0.19}^{+0.21}$ & $505_{-179}^{+519}$ & $36.2 / 57$ & KW+BAT \\
$(23.497,38.217)$ & $\mathrm{CPL}$ & $-1.07_{-0.42}^{+0.56}$ & $457(>193)$ & $46.1 / 57$ & KW+BAT \\
$(38.217,49.993)$ & $\mathrm{CPL}$ & $-1.31_{-0.16}^{+0.19}$ & $783(>362)$ & $67.1 / 57$ & KW+BAT \\
$(49.993,76.489)$ & $\mathrm{CPL}$ & $-1.33_{-0.21}^{+0.27}$ & $643(>246)$ & $56.7 / 57$ & KW+BAT \\
$(-8.887,76.489)$ & $\mathrm{CPL}$ & $-1.12_{-0.09}^{+0.09}$ & $523_{-118}^{+198}$ & $49 / 57$ & KW+BAT \\
\hline & & & & &
\end{tabular}

Notes.

a Time-resolved spectral parameters for joint fits on Konus-Wind and Swift-BAT data for the first emission episode of GRB 091024A. Uncertainties are approximated with the bootstrap method and given to the $1 \sigma$ confidence level.

$\mathrm{b}$ Time intervals derived with a Bayesian Block technique from the Konus-Wind light curve. Times are relative to the Swift-BAT trigger.

\subsection{Energetics}

Using the durations and spectra derived from $\mathrm{KW}$, we calculate the fluence and isotropic energy radiated in $\gamma$-rays. The total rest-frame $1-10^{4} \mathrm{keV}$ isotropic equivalent radiated energy from all three emission episodes is $E_{\gamma, \text { iso }} \approx(4.5 \pm$ $0.09) \times 10^{53} \mathrm{erg}$, with $0.90_{-0.03}^{+0.04}, 0.50_{-0.05}^{+0.04}$, and $3.1_{-0.06}^{+0.07} \times 10^{53} \mathrm{erg}$ corresponding to each episode, and we find that all three emission episodes fall within the $2 \sigma$ region of the Amati relation (Amati et al. 2002). The interpulse segment also emits low-level emission, which when integrated over its long duration radiates an additional $0.74_{-0.04}^{+0.07} \times 10^{53} \mathrm{erg}$, bringing the total radiated energy up to $5.2_{-0.09}^{+0.12} \times 10^{53} \mathrm{erg}$.

Deriving the kinetic energy (KE) contained in the GRB ejecta is a more involved process requiring more detailed broadband afterglow modeling (Panaitescu \& Kumar 2001) or X-ray afterglow data (Freedman \& Waxman 2001; Berger et al. 2003; Lloyd-Ronning \& Zhang 2004; Zhang et al. 2007). Following the theory proposed by Zhang et al. (2007), we determine the spectral regime of the X-ray afterglow and calculate the $\mathrm{KE}$ and radiative efficiency, $E_{K}$ and $\eta$, where $\eta=E_{\gamma \text {,radiated }} / E_{\text {total }}$.

A main ingredient in this analysis is the X-ray flux. Simply extrapolating the observed X-ray light curve back to early times causes the fit to significantly overestimate the amount of flux at early times compared to what is expected from the BAT emission. We therefore consider two different smoothed broken power law fits which give a more realistic prediction of the level of early-time flux. The best-fit parameters are $\alpha_{1}=1.06 \pm 0.37$, $\alpha_{2}=2.39 \pm 0.58$, and $t_{\text {break }}=9.57 \times 10^{3} \mathrm{~s}$ with smoothing parameter $s=-3$. This fit still slightly overestimates the expected emission when extrapolating into the BAT band, and we take an approximate $\alpha_{1} \approx 0.8 \pm 0.1$ with identical $t_{\text {break }}$ and $\alpha_{2}$ for our best intuitive guess of the trend of the early X-ray afterglow. We do not claim that this simplistic scenario is the true shape of the light curve but adopt it as a guide for the subsequent efficiency calculation.

Next, we use the light curve and spectral fits of the X-ray data and the predicted standard model closure relations to ascertain the spectral regime, $v_{m}<v_{X}<v_{c}$ or $v_{X}>\max \left[v_{m}, v_{c}\right]$. We use the shallow light curve slope for the early X-ray light curve, $\alpha_{1} \approx 0.8 \pm 0.1$, so as to not overestimate the amount of flux and to provide a lower limit on $E_{K}$. The slopes are consistent with the constant-density interstellar medium (ISM) model in the
$v_{m}<v_{X}<v_{c}$ regime over a large range of $p$ values, or ISM and wind models in the $v_{X}>\max \left[v_{m}, v_{c}\right]$ regime with $1<p<2$. The very shallow spectral slope of $\beta=0.49$, however, gives a very low value of $p$ in the latter spectral regime and we therefore assume that the X-ray data lie in the $v_{m}<v_{X}<v_{c}$ regime. This is consistent with the treatment of shallow $\beta$ by Zhang et al. (2007) and implies $p=1.98_{-0.21}^{+0.23}$. This is not the preferred spectral regime to determine $E_{K}$ as it is dependent more heavily on the value of $\epsilon_{B} . E_{K}$ in units of $10^{52} \mathrm{erg}$ is expressed by Zhang et al. (2007, their Equation (13)) for this spectral regime as

$$
\begin{aligned}
E_{K, 52}= & {\left[\frac{v F_{v}\left(v=10^{18} \mathrm{~Hz}\right)}{6.5 \times 10^{-13} \mathrm{erg} \mathrm{cm}^{-2} \mathrm{~s}^{-1}}\right]^{4 /(p+3)} } \\
& \times D_{28}^{8 /(p+3)}(1+z)^{-1} t_{d}^{3(p-1) /(p+3)} \\
& \times f_{p}^{-4 /(p+3)} \epsilon_{B,-2}^{-(p+1) /(p+3)} \epsilon_{e,-1}^{4(1-p) /(p+3)} \\
& \times n^{-2 /(p+3)} v_{18}^{2(p-3) /(p+3)},
\end{aligned}
$$

where $v F_{v}$ is the flux in the X-ray band, $D_{28}$ is the luminosity distance in units of $10^{28} \mathrm{~cm}, n$ is the ambient density, $v_{18}$ the observed band in units of $10^{18} \mathrm{~Hz}$, and $f_{p}$ is a function of $p$ defined as (Equation (10) of Zhang et al. 2007)

$$
f_{p}=6.73\left(\frac{p-2}{p-1}\right)^{(p-1)}\left(3.3 \times 10^{-6}\right)^{(p-2.3) / 2} .
$$

The radiative efficiency of a burst is assumed to be a constant value but the choice of time for calculating the efficiency, $t_{d}$, varies among bursts. We take $t_{d}$ to be the time of the second optical peak, $\sim 0.03$ days, as we are exploring the possibility that this is either approximately the deceleration time or the end of the energy injection from the central engine. In both cases we want to make sure that there is no significant further addition of energy that will skew the results of the calculation. We use Equation (1) with $\epsilon_{B}=0.001, \epsilon_{e}=0.1, n=1, p=2.1$, and $v F_{v}=4 \times 10^{-10} \mathrm{erg} \mathrm{cm}^{-2} \mathrm{~s}^{-1}$ at the assumed $t_{d}$ of $\sim 0.03$ days, and we find a conservative lower limit of $\sim 2 \times 10^{53} \mathrm{erg}$ for $E_{K}$, which implies $\eta \approx 0.4$, or a relatively inefficient radiator. The radiative efficiency is also dependent on the value of $p$ and changes dramatically due to the functional form of $f_{p}$. The value of $p=2.1$ roughly maximizes Equation (2) and changes to this value will results only in increases of the KE. For the case where $p \approx 3$, which becomes important for the late-time afterglow modeling, the efficiency drops to the order of $\eta \approx 0.2$. Decreasing $\epsilon_{B}$ or $n$ increases the amount of KE, further lowering the value of the radiative efficiency. Zhang et al. (2007) find that for other bursts in this spectral regime, $\epsilon_{B}$ is generally very low $\left(<10^{-4}\right)$ to satisfy $v_{c}>v_{X}$, and could further justify an increase in the estimated KE of GRB 091024A. The value of the efficiency is only an estimate, but within the relatively broad range of parameters for this burst we have established the presence of a significant amount of KE available to power the observed structure of the optical afterglow.

\section{ORIGIN OF THE OPTICAL EMISSION}

GRBs for which early optical emission is observed show a range of properties. Some exhibit clear temporal coincidence between optical and $\gamma$-ray features, suggesting a prompt origin (Vestrand et al. 2005, 2006; Racusin et al. 2008; Guidorzi et al. 2011a; Kopač et al. 2013). Others show single peaks or power law decays consistent with the onset or continuation of the 
afterglow (e.g., Akerlof et al. 1999; Molinari et al. 2007; Page et al. 2007; Melandri et al. 2008). GRB 091024A has multiple peaks in the $\gamma$-ray and optical bands (Figure 5).

We use a number of cross-correlation tests to see if the optical emission shows any temporal correlation with the $\gamma$-rays. For every step of the KW light curve (2.944 s), we shifted the KW curves along a range of temporal intervals, from -2000 to +2000 s. After shifting, we rebinned the KW curves so as to match the optical binning as closely as possible, and then calculated the Pearson, Spearman, and Kendall correlation coefficients of the optical flux versus $\gamma$-ray rates. For 1401 trial lags the coefficients are 0.71 (lag $=-490 \mathrm{~s}$ ), 0.78 $(\mathrm{lag}=-230 \mathrm{~s})$, and $0.62(\mathrm{lag}=-230 \mathrm{~s})$, respectively, where the lag corresponds to the temporal shift of the KW light curve with respect to the optical light curve. The associated probabilities for these coefficients are of the range of $10^{-3}-10^{-4}$ and correspond to the alignment of the second and third $\gamma$-ray peak with the first optical peak (see Figure 4). We also stretched the $\gamma$-ray light curve by a scale factor and performed a similar cross-correlation. We conclude that, despite the richness of temporal structure and significant overlap in the $\gamma$-ray and optical light curves, there is no correlation (or anticorrelation) between the prompt $\gamma$ ray emission and the observed optical peaks. This implies that the optical emission is from a distinct physical process from the prompt $\gamma$-rays and likely consistent with an external-shock origin, as detailed below.

Further justification for the external-origin hypothesis comes from the morphology of the first optical peak. A diagnostic of possible internal optical emission is the pulse-width $/ t_{\text {peak }}$ ratio (e.g., Kopač et al. 2013), with GRB 091024A showing a value $>1$, larger than the typical internal ratio of $<1$. Alternatively, if the optical peaks are due solely to forward-shock (FS) emission, they show consistency with the empirically fit rise and decay slopes of other bursts $\left(\alpha_{\text {rise }} \approx-0.3\right.$ to -4 and $\left.\alpha_{\text {decay }} \approx 0.6-1.8\right)$ and the empirical $L-t_{p}$ and $L-E_{\gamma \text {,iso }}$ relations presented by Liang et al. (2013).

\subsection{Afterglow Modeling}

Having excluded a prompt origin for the optical emission, we now fit the optical light curve with several external synchrotron shock models to determine the nature of the optical peaks, which we conclude are consistent with the presence of strong reverse-shock (RS) and FS components as well as late-time rebrightening. We show that the emission is consistent with an intermediate shell-thickness regime which can explain the shallow rising light curve at early times, which is a natural consequence of the central engine not ceasing after the first $\gamma$-ray emission episode but continuing to emit at a low level.

\subsubsection{Forward Shock and Refreshed Shock}

Visual inspection of the optical afterglow shows that the first optical peak occurs before the second and third $\gamma$-ray emission episodes (Figure 4). It would be reasonable, then, to expect that the first optical peak evolves like a single GRB with the characteristics of the first emission episode without yet being affected by later emission. To this end, we model the early optical afterglow with the parameters of the first $\gamma$-ray episode: $E_{\text {iso }} \approx 1 \times 10^{53} \mathrm{erg}$, duration (from $\mathrm{KW}$ ) $T=88 \mathrm{~s}$. In this scenario, the first peak is caused by the decelerating fireball from the first emission episode while the second peak is a rebrightening feature caused by the $\mathrm{KE}$ injected into the blastwave from the second and third $\gamma$-ray episodes. Depending on the burst parameters, specifically the unknown values of the

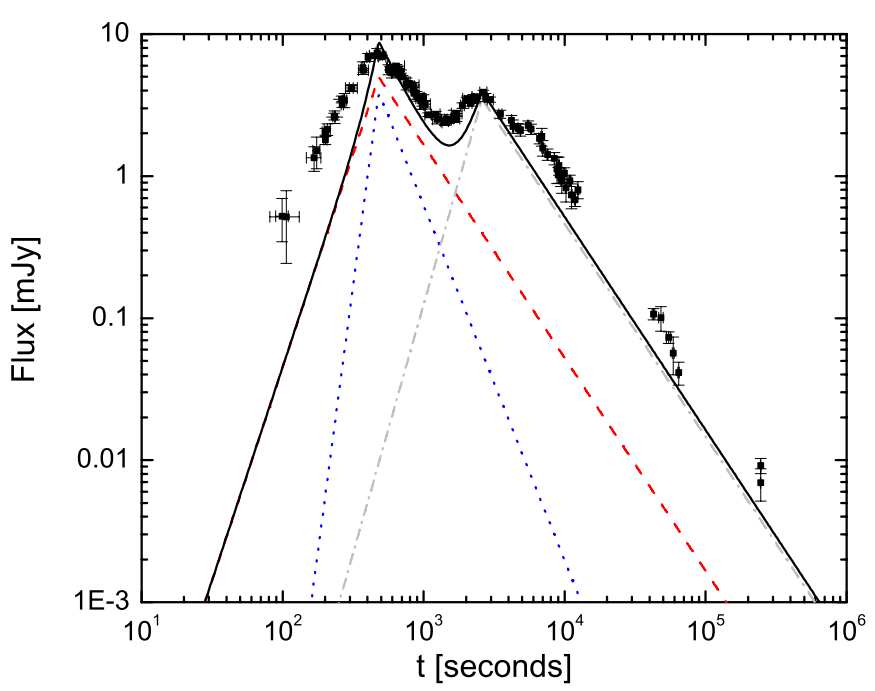

Figure 8. Afterglow modeling of GRB 091024A as a forward-shock peak (red; dashed) with simultaneous reverse shock (blue; dotted) followed by a rebrightening peak (gray; dash-dot). The sum of the components is in black (solid). $F_{v, \max , f}=5 \mathrm{mJy}, t_{p, f}=t_{p, r}=480 \mathrm{~s}, F_{v, \max }$ rebrightening $=3.5$ $\mathrm{mJy}, t_{p, \text { rebrightening }}=2600 \mathrm{~s}, p=3$.

(A color version of this figure is available in the online journal.)

microphysical parameters, the typical frequency of the FS, $v_{m, f}$, will fall at or around the optical band, $v_{O}$. In this simple model, if $v_{m, f}$ falls at or below the observed band the onset of the deceleration of the fireball and the RS crossing time will occur simultaneously.

In Figure 8 we show the standard evolution of the FS with a maximal flux from the optical light curve $\left(t^{3}\right.$ rise, $t^{-3(p-1) / 4}$ decay) peaking at $5.5 \mathrm{mJy}$ combined with the expected RS emission and a rebrightening feature. We have modeled the latter as a broken power law peaking at $3.5 \mathrm{mJy}$, rising as $t^{3.5}$, and decaying with the same slope as the FS, $t^{-3(p-1) / 4}$. The observed late-time slope of $\sim 1.5$ implies $p \approx 3$ and we assume a constant ISM density of 1 proton $\mathrm{cm}^{-3}$. Reducing $p$ (e.g., $p \approx 2.2-2.6)$ increases the amount of RS flux and makes the slope of the FS and of the rebrightening feature more shallow. Reducing the peak flux of the FS with this lower $p$ causes the first peak to decrease but also lowers the contribution after the peak, causing the model to further underpredict between the two optical peaks. Additionally, the shallower slope is inconsistent with the late-time optical data, requiring a mechanism to reduce the late-time flux (e.g., a jet break).

As discussed in Section 3.3, the fireball is likely an inefficient radiator, leaving an ample supply of $\mathrm{KE}$ from the central-engine activity that powered the later $\gamma$-ray emission episodes to refresh the blastwave and cause the rebrightening feature. The ratio of radiated energy from the combined second and third emission episodes to the first peak is about 3.5, which should approximate the ratio of $\mathrm{KE}$ deposited under the assumption that the $\mathrm{KE}$

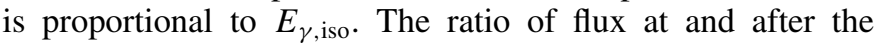
rebrightening peak in Figure 8 is about a factor of $\sim 8$ above the blastwave solution, which is above the simplistic estimate of the amount of energy injected by the later central-engine activity. This model has difficulty in explaining the late-time structure of the light curve (i.e., the small rebrightening at $T \approx 5000 \mathrm{~s}$ ), especially if a significant amount of $\mathrm{KE}$ is needed to explain the flux of the two large blastwave features.

The early slope of the first peak is not well modeled in either case. Invoking an intermediate ambient medium density profile 
can reduce the slope, but there is no clear physical interpretation for such a density profile; furthermore, it affects the afterglow evolution after the crossing of $v_{m}$ (Liang et al. 2013). This evidence, in conjunction with the shortfalls discussed above, leads us to consider our next case, that the first optical peak is produced from a RS and the second by the FS.

\subsubsection{Reverse Shock and Forward Shock}

In this interpretation, we assume that the first optical peak is caused by the external/RS and the second is caused by the FS emission as the typical frequency of the FS, $v_{m, f}$, crosses the observed band, $v_{R}$. A prominent RS has been theoretically predicted (Akerlof et al. 1999; Sari \& Piran 1999; Mészáros \& Rees 1999) and seen in a handful of GRBs (Kulkarni et al. 1999; Kobayashi \& Sari 2000; Gomboc et al. 2008; Gendre et al. 2010; Laskar et al. 2013). Using the same parameters for the first $\gamma$-ray episode as above, we model the afterglow with the theoretical predictions of these two components. Due to the well-sampled optical light curve, we have both the observed times and maximal fluxes of both shock components, which add important constraints that are often missing in this type of analysis. Similarly to the previous section, the FS evolves as $t^{3}$ at very early times, $t^{1 / 2}$ as the typical FS frequency approaches the optical band, and then decays as $t^{-3(p-1) / 4}$ as the fireball decelerates.

Depending on the value of the critical Lorentz factor, $\gamma_{\text {crit }}=$ $\left(3 E / 32 \pi n m_{p} c^{2} \Delta_{0}^{3}\right)^{1 / 8}$, the RS can (thick shell case, $\left.\gamma_{0}>\gamma_{\text {crit }}\right)$ or cannot (thin shell case, $\gamma_{0}<\gamma_{\text {crit }}$ ) effectively decelerate the unshocked shell material (Kobayashi \& Sari 2000; Kobayashi \& Zhang 2003; Zhang et al. 2003; Zhang \& Kobayashi 2005). Here, $\gamma_{0}$ is the initial Lorentz factor of the shell, $m_{p}$ is the proton mass, and $\Delta_{0}$ is the initial shell width, which in the internalshock model is approximated by the intrinsic duration of the $\mathrm{GRB}, \sim c T /(1+z)$ (Kobayashi et al. 1997). These cases are the extreme scenarios of relativistic or Newtonian RS, and they provide clear predictions for the rising slope of the RS: $t^{0.5}$ for thick shell (relativistic) and $t^{3 p-3 / 2} \approx t^{5-6}$ for thin shell (Newtonian).

In the case of GRB 091024A, we see an intermediate rising slope of $\sim 2.3$, which clearly deviates from the predictions. Nakar \& Piran (2004) showed that if a burst falls into an intermediate regime between fully relativistic and Newtonian, the rising slope of the RS can change dramatically, characterized by the dimensionless parameter $\xi=\left(l / \Delta_{0}\right)^{1 / 2} \gamma_{0}^{-4 / 3}$, where $l=\left[3 E /\left(4 \pi n m_{p} c^{2}\right)\right]^{1 / 3}, \Delta_{0}$ is the shell width, and $\gamma_{0}$ is the initial Lorentz factor. The cases $\xi \ll 1$ and $\gg 1$ correspond to relativistic and Newtonian, respectively. An intermediateregime fireball would require longer-lasting central-engine activity, which is justified in the case of GRB 091024A since we see low-level emission between the first two $\gamma$-ray episodes (see Section 3.2.2).

Having observed the rise of the RS $\left(\alpha_{\text {rise }} \approx-2.3\right.$ ), we can estimate the value of $\xi$ from the numerical results of Nakar \& Piran (2004):

$$
\alpha_{\text {rise }} \approx N_{\alpha}\left[0.5+\frac{p}{2}\left(\xi-0.07 \xi^{2}\right)\right],
$$

where $N_{\alpha}$ is a numerical constant $=1.2$. Depending on the value of $p, \xi$ can range from $\sim 1$ to 1.4 ; it is consistent with the intermediate-regime treatment where $\xi \approx 1$.

In a complementary analysis, Harrison \& Kobayashi (2013, hereafter HK13) used hydrodynamical simulations to study afterglows with significant external/RS emission and found that previous treatments significantly underestimate the amount of magnetization in the RS by as much as two orders of magnitude, especially in intermediate regions where $\xi \approx 1$. Having larger magnetization in the RS significantly lowers the initial Lorentz factor, helping to move the RS from a highly relativistic (thick shell) regime that significantly decreases the RS slope before the RS crossing time. The inclusion of magnetization is further rationalized by the discovery of $10 \%$ polarization in the early afterglow of GRB 090102 (Steele et al. 2009), proving the existence of large-scale ordered magnetic fields in that, and likely other, bursts. HK13 provide updated magnetization expressions based on their hydrodynamical simulations with numerical corrections to the theoretical framework of Zhang et al. (2003) and Zhang \& Kobayashi (2005), and to the treatment of GRB 061126 by Gomboc et al. (2008).

Since the duration of the central-engine activity is longer than simply the observed initial $\gamma$-ray episode, we can estimate the duration of the central engine needed to create the observed shallow slope from

$$
T \approx t_{p, r}\left(\frac{\xi^{2}}{5}+1\right)^{-1}
$$

where $t_{p, r}$ is the peak time of the RS and $T$ is the duration of the emission. Another consequence of the intermediate regime is the estimation of the initial Lorentz factor, $\gamma_{0}$. In the thin shell regime, we can estimate $\gamma_{0}$ from the crossing time of the shell, $t_{x}$. We observe this peak at $480 \pm 19 \mathrm{~s}$ from the onset of the GRB, and theoretically it should occur at $t_{x} \approx\left(\gamma_{x} / \gamma_{\text {crit }}\right)^{-8 / 3} T$, where $\gamma_{x}$ is the Lorentz factor at the shock-crossing time, $\sim \operatorname{minutes}\left[\gamma_{0}, \gamma_{\text {crit }}\right]$ (Sari \& Piran 1999; Kobayashi \& Sari 2000; Zhang et al. 2003). The correction factor to the deceleration time in the thin shell regime from HK13 is simply $C_{t}=2^{-4 / 3} x^{-8 / 3}$, where $x=\gamma_{d} / \gamma_{0}$, the ratio of the Lorentz factor of the shocked shell material relative to the unshocked shell and the initial Lorentz factor. The value of $x$ in turn depends on $\xi$ as

$$
\xi^{2} \approx \frac{24 x^{8 / 3}}{2^{2 / 3}\left(1-x^{2}\right)\left(2+3 x+2 x^{2}\right)} .
$$

Next, we can estimate the magnetization parameter

$$
R_{B}=\left(\frac{R_{F}^{3} \gamma_{0}^{4 \alpha-7}}{C_{F}^{3} C_{m}^{2(\alpha-1)} R_{t}^{3(\alpha-1)}}\right)^{2 /(2 \alpha+1)}
$$

the typical frequency of the RS

$$
v_{m, r}\left(t_{x}\right) \approx C_{m} \gamma_{0}^{-2} R_{B}^{1 / 2} v_{m, f}\left(t_{x}\right),
$$

and the maximal flux of the RS at the peak time and at the typical frequency

$$
F_{v, \max , r}\left(t_{x}\right) \approx C_{F} \gamma_{0} R_{B}^{1 / 2} F_{\nu, \max , f}
$$

(Kobayashi \& Zhang 2003; Zhang et al. 2003; Zhang \& Kobayashi 2005; HK13), where $C_{F}$ and $C_{m}$ are the numerical correction factors from HK13, $\alpha=(3 p+1) / 4$ is the decay slope of the RS, $R_{t}=t_{p, \mathrm{FS}} / t_{p, \mathrm{RS}}$ is the ratio of the FS peak time to RS peak time, and $R_{F}=F_{v, \max , \mathrm{RS}} / F_{v, \max , \mathrm{FS}}$ is the ratio of the RS peak flux and FS peak flux (Gomboc et al. 2008; HK13). The values of $R_{t}$ and $R_{F}$ can be measured directly from the optical light curve. 

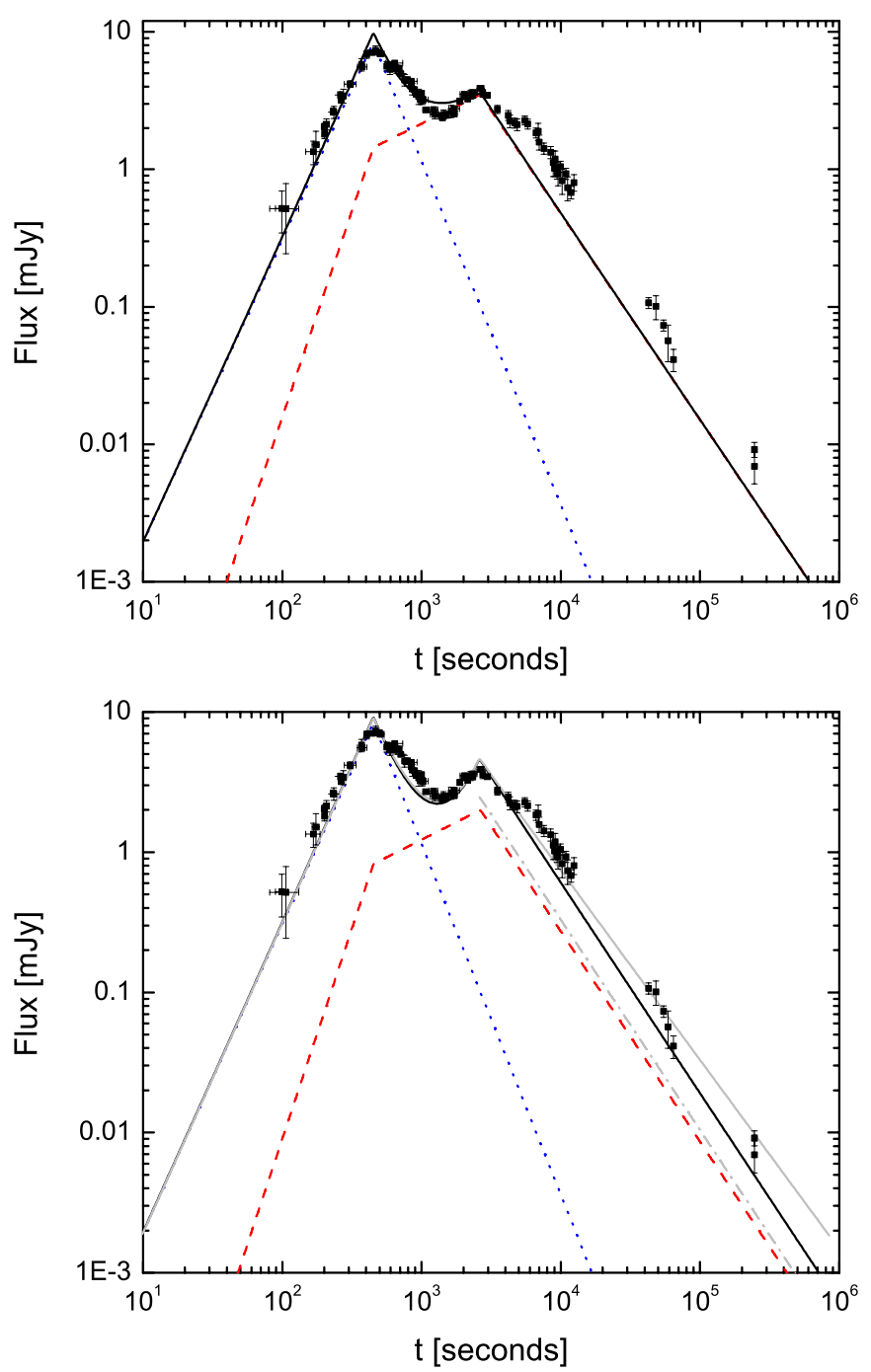

Figure 9. Afterglow modeling of the optical light curve of GRB 091024A assuming that the first optical peak is due to emission from a magnetized reverse shock (blue; dotted) in an intermediate regime between the thin and thick shell approximations, and that the second peak is either the forward shock (red; dashed) or forward shock and simultaneous rebrightening (gray; dash-dot). The sum of all components is given in black (solid). (a) $F_{v, \max , f}=3.5 \mathrm{mJy}$, $p=3$ (b) $F_{v, \max , f}=2 \mathrm{mJy}$ and $F_{v, \text { max, rebrightening }}=2.5 \mathrm{mJy}, p=3$ (solid; black) and $p=2.8$ (solid, gray).

(A color version of this figure is available in the online journal.)

Figure 9 shows two examples of model fits to the optical light curve of GRB 091024A. Using the RS rise slope we calculate $\xi \approx 0.95$ for $p=3$. The first panel shows the model predictions using the observed parameters $t_{x}=t_{m, r}=480 \mathrm{~s}$, $F_{v, m, r} \approx 7.5 \mathrm{mJy}, F_{v, m, f} \approx 3.5 \mathrm{mJy}, t_{p, f} \approx 0.03$ days, and $n_{0}=1$ proton $\mathrm{cm}^{-3}$. This implies $\epsilon_{e} \approx 0.18, \gamma \approx 115$, $R_{B} \approx 100$, and $T \approx 380 \mathrm{~s}$. In addition, the rising and decay slopes of the RS better approximate the observed light curve. This model, however, washes out some of the structure between the two optical peaks, due to the $t^{1 / 2}$ component of the FS emission before the peak, and is problematic from an energetics point of view since the modeling assumes that the driving force of the light curve is only the first $\gamma$-ray emission episode. If a significant fraction of the KE emitted by the burst is yet to be deposited into the blastwave, this solution does not provide the flexibility for energy injection into the blastwave, apart from the small feature at $\sim 5000 \mathrm{~s}$.
In order to address this problem, we add a rebrightening component near the time of the second optical peak. In this case, the second optical peak will be a superposition of the two components, allowing us to decrease the importance of the FS flux and see the effect on the modeled light curve (Figure 9, panel (b)). Lowering the peak flux of the FS increases the magnetization parameter but only marginally affects the RS peak flux. Similar to the FS analysis, we introduce a rebrightening component that evolves as $t^{3.5}$ and $t^{-3(p+1) / 4}$ and peaks near the FS peak time of $2600 \mathrm{~s}$. We model the RS and FS as discussed above, with the exception of varying the peak flux of the FS. The second panel of Figure 9 shows a model fit with the same $\xi$ and $p$, a FS peaking at $2 \mathrm{mJy}$ and the rebrightening feature at 2.5 mJy, implying $\epsilon_{e} \approx 0.18, \gamma \approx 115, R_{B} \approx 185$, and $T \approx 380 \mathrm{~s}$. This also adds flexibility for the energy injection in the form of the rebrightening peak, which is comparable to the FS emission, still leaving room for further injection of energy to create the feature at $5000 \mathrm{~s}$. The ratio of flux from the total solution to the FS solution is about a factor of 2.3. Decreasing the value of $p$ slightly shallows all the decay slopes, but $p$ less than $\sim 2.8$ begins to exceed the flux of the late-time data points. With a $\xi=1.03$, this implies $\epsilon_{e} \approx 0.21$, and it has the additional effect of decreasing the magnetization and duration to $\sim 130$ and $370 \mathrm{~s}$, respectively.

The behavior of the FS evolution at very early times (before the peak time) in this intermediate model is not well understood. If it behaves similarly to the RS in that the slope lies somewhere between the extreme solutions of the thin and thick shell cases, it is possible that the FS slope is shallower than the expected $t^{3}$ evolution in the thin shell regime. Decreasing the FS slope to $\sim 1$ has only a small effect on the total flux of the light curve, and the behavior lies within the error bars of the observed data points.

\section{ULTRA-LONG GRB SAMPLE}

GRB 091024A is one of a handful of bursts with interesting and very long duration prompt $\gamma$-rays. Its prompt emission has strong (i.e., high flux) $\gamma$-ray emission totaling $\sim 700 \mathrm{~s}$ that is interrupted by long periods of low, almost background-level emission. In addition to its prompt $\gamma$-ray emission, this burst has unique and well-sampled optical emission showing multiple peaks and bumps that we have interpreted as emission from a magnetized external/RS followed by an FS with significant energy injection.

Next, we review other ultra-long GRBs with $\gamma$-ray duration $\gtrsim 1000 \mathrm{~s}$ discussed in the literature and present them grouped by similar overarching themes and characteristics in their $\gamma$ ray emission. All of these bursts show $\gamma$-ray emission at times beyond what is expected for most GRBs ( $\gtrsim 1000 \mathrm{~s}$ ), whose distribution of $t_{90}$ durations peaks at a few tens of seconds and extends to a few hundred seconds, depending on the energy range of the detector (Virgili et al. 2012; Qin et al. 2013). A summary of relevant observations is found in Table 6. Although a number of possible very long duration events were discovered with $C G R O /$ BATSE (Tikhomirova \& Stern 2005), we do not include these due to the inability to robustly associate the many $\gamma$-ray episodes with one GRB (Pal'shin et al. 2012).

\subsection{Interrupted Emission}

GRBs within this category have episodes of prompt $\gamma$-ray emission separated by long stretches of low-level or quiescent activity lasting hundreds of seconds that require vast reductions 
Table 6

Ultra-long GRB Sample

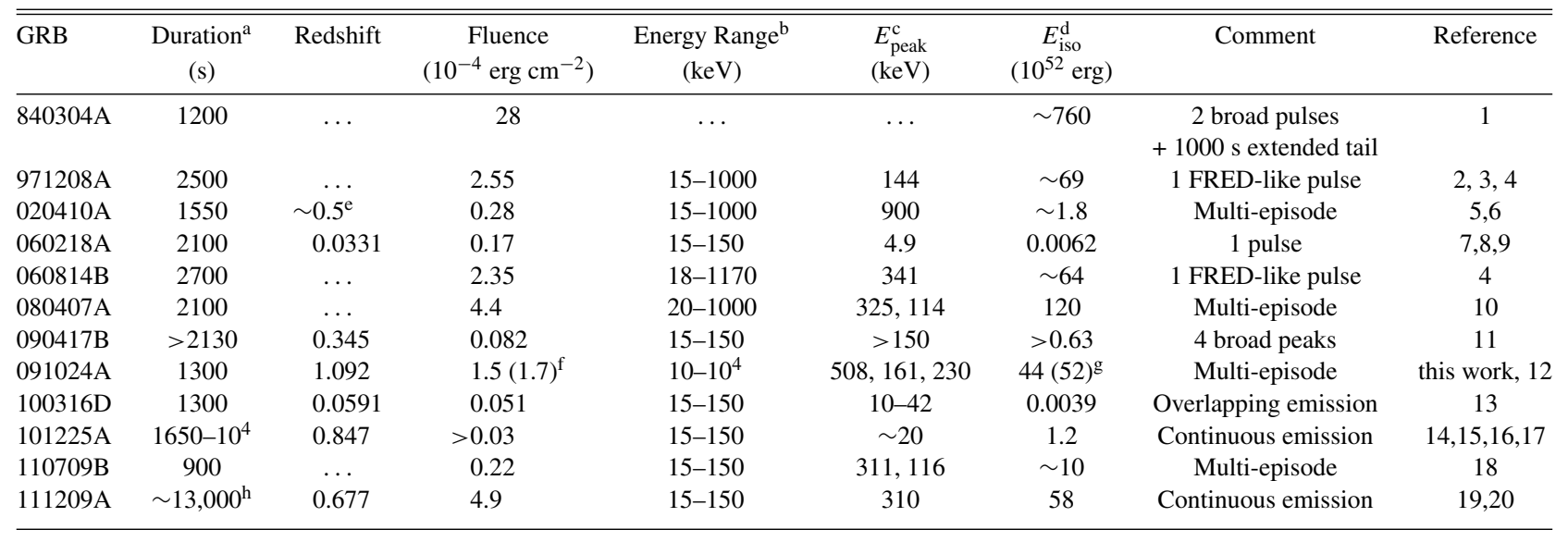

Notes.

a Approximate duration of the entire $\gamma$-ray interval, including intervals of low-level or quiescent emission.

$\mathrm{b}$ Energy range in which the fluence was reported.

${ }^{c}$ Lines with multiple entries denote the peak energy of each emission episode.

${ }^{\mathrm{d}}$ For bursts with unknown redshift, the radiated isotropic equivalent energy is estimated at $z=1$.

e Levan et al. (2005) find this estimate for the redshift from modeling and fitting of the late-time light curve with expected supernova emission. Nicastro et al. (2004) provide an estimate of $0.9<z<1.5$ from the Amati relation.

${ }^{\mathrm{f}}$ Second value is the total fluence including the interpulse region between the first and second $\gamma$-ray episodes.

$\mathrm{g}$ Second value includes the fluence contribution from the interpulse region.

${ }^{\mathrm{h}}$ We estimate the $\gamma$-ray duration to be consistent in our burst selection. Gendre et al. (2013) report a duration that includes the longer-lasting X-ray emission attributed to the central engine, increasing their value to $\sim 25 \mathrm{ks}$.

References. (1) Klebesadel et al. 1984; (2) Connaughton et al. 1997; (3) Giblin et al. 2002; (4) Pal'shin et al. 2008; (5) Nicastro et al. 2004; (6) Levan et al. 2005; (7) Campana et al. 2006; (8) Soderberg et al. 2006; (9) Liang et al. 2006b (10) Pal'shin et al. 2012; (11) Holland et al. 2010; (12) Gruber et al. 2011; (13) Starling et al. 2011 (14) Racusin et al. 2011; (15) Campana et al. 2011; (16) Thöne et al. 2011; (17) Levan et al. 2013 (18) Zhang et al. 2012; (19) Gendre et al. 2013; (20) Stratta et al. 2013.

in central-engine activity. Bursts that show this behavior are: GRB 080407A, GRB 091024A, GRB 110709B (see the Appendix for full details). Additionally, most of the BATSE bursts presented by Tikhomirova \& Stern (2005), assuming the emission episodes could be linked to the same GRB, have this type of behavior (durations of 500-1300 s).

Even with a smaller sample size than the other category of bursts, there is still a range of afterglow properties. GRB 091024A and GRB 110709B both show very high flux $\gamma$-ray emission and normal to weak X-ray afterglows. We have previously discussed the optical properties of GRB 091024A, and GRB 110709B is classified as a dark burst.

In this case, "duration" has less significance than in the continual emission case. Even if the central engine has not completely ceased to emit, as we have shown in the spectral analysis of GRB 091024A, the large interpulse interval could change, for example, the evolution of the expected afterglow emission. This is exemplified in GRB 091024A, whose intermediate thin/thick shell regime modifies the evolution of the $\mathrm{RS}$ emission. It might also provide insight into the distribution of matter in the accretion disk or how matter is fed into the central engine (e.g., a fallback accretion model; Wu et al. 2013 and references therein). Long quiescent times have also been interpreted in the literature as outliers to the log-normal distribution of interpulse times, possibly indicating a different mechanism than other intervals (Quilligan et al. 2002; Nakar \& Piran 2002; Drago \& Pagliara 2007). There is also weak evidence for a correlation between the pulse width and the following interval (Nakar \& Piran 2002). Alternatives include the formation of quark phases (Drago et al. 2008) or changes to the distribution of ejected shells from the central engine (e.g., Ramirez-Ruiz et al. 2001).
GRBs 080407A, 091024A, and 110709B have long interpulse regions lasting $\sim 1400,500$, and $600 \mathrm{~s}$, respectively. The BATSE bursts discussed previously have interpulse episodes of a similar timescale, with a few bursts reaching 600-1400 s. All bursts tend to have emission episode durations of 50-200 s. An intermediate case is found in bursts like GRB 010619A, discovered by BeppoSAX (Frontera et al. 2009; Guidorzi et al. 2011a). Its duration is shorter than the rest of the sample $\left(T_{90} \approx 450 \mathrm{~s}\right)$, but it shows many of the characteristics of this class, including intense emission episodes and significant periods of quiescence between emission episodes. This behavior is mimicked in various other bursts, e.g., GRBs 001213A and 121217A.

Previous works have not discussed the implications of selection bias on ultra-long GRBs with interrupted emission. In this case $t_{90}$ is not as meaningful, since the emission drops to nearly background level between emission episodes. In the case of GRB 091024A, it could be argued that a $1200 \mathrm{~s}$ "duration" is misleading since more than half of that time is nearbackground emission or quiescence. Other properties, like the peak flux, are also different in bursts with continuous emission. Instead of low-level continuous emission peaking on the order of $0.1-0.5 \mathrm{ph} \mathrm{cm}^{-2} \mathrm{~s}^{-1}$ for Swift-detected bursts, these have emission episodes that range from $50 \mathrm{~s}$ to $200 \mathrm{~s}$ and peak fluxes of $\sim 10-1000$ photon $\mathrm{cm}^{-2} \mathrm{~s}^{-1}$. When the individual emission episodes are plotted on the fluence- $t_{90}$ and $1-\mathrm{s}$ peak flux $-t_{90}$ planes, they are more consistent with the population of "normal" long-duration GRBs, increasing the chances that they will be detected (see Figure 10). Biases in the detectability of all the associated emission episodes now become important. In the four post-BATSE bursts that show interrupted emission, the first emission episode is either comparable to or brighter than 

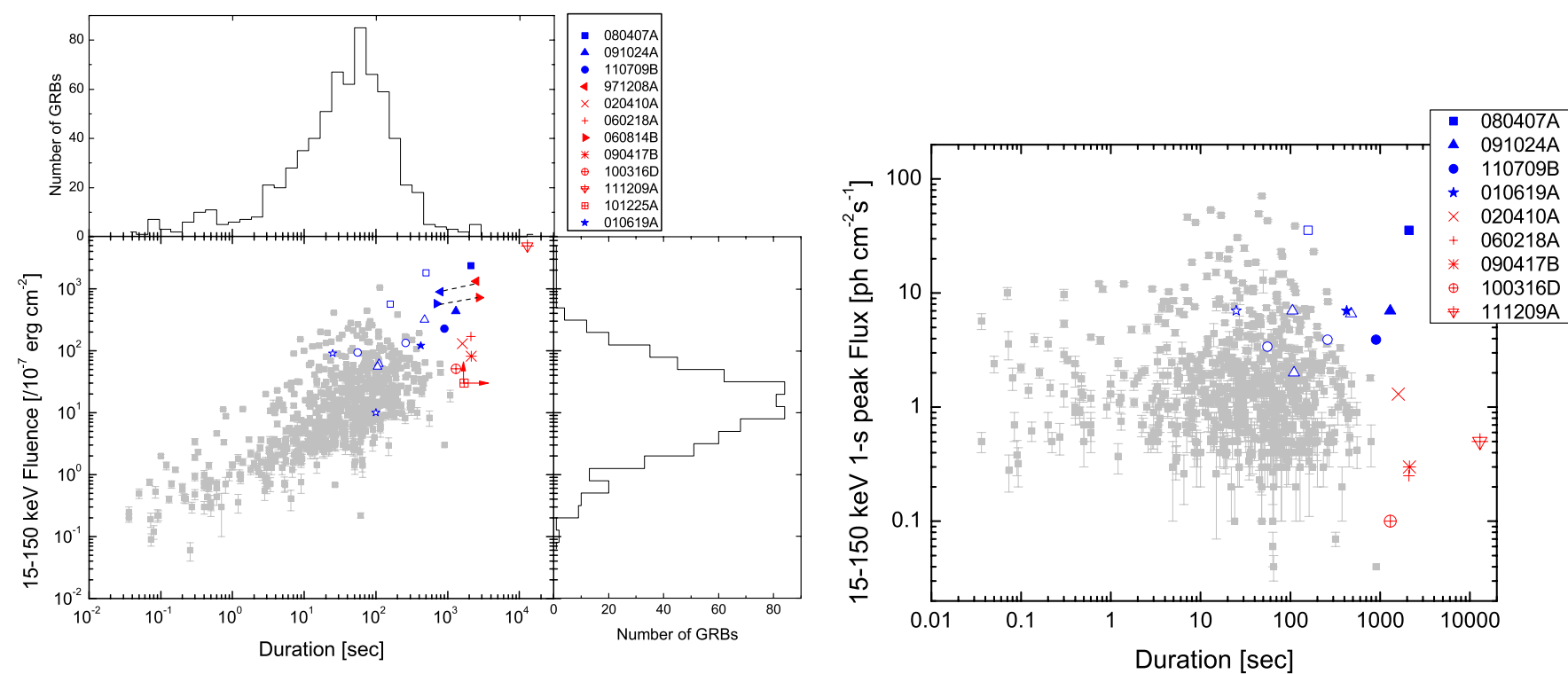

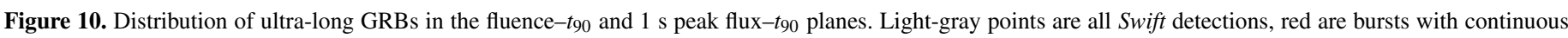

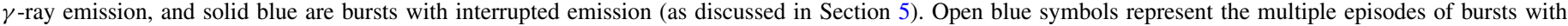

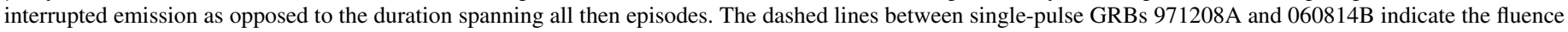

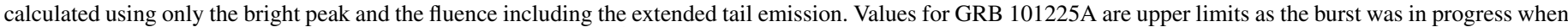
it entered the Swift field of view. The true duration could be $\gtrsim 10^{4} \mathrm{~s}$.

(A color version of this figure is available in the online journal.)

the subsequent peaks. This is exemplified by GRBs 091024A and 110709B that triggered Fermi-GBM and Swift, respectively, on both their early and late emission episodes. The collection of BATSE bursts show similar behavior although not as pronounced.

\subsection{Continuous Emission}

This subset of ultra-long GRBs shows long-lasting continual emission from the central engine manifested as a single fast-rise-exponential-decay-(FRED)-like pulse or one or more broad overlapping pulses. These include GRBs 840304A, 971208A, 060218A, 060814B, 090417B, 100316D, 101225A, and 111209A (see the Appendix for full details). A unifying characteristic of this group is their long-lasting but very low level emission, as highlighted in Figure 10. This weak emission is likely the greatest limitation to the detection of this type of very long event, which inhibits their observation at high redshift and is likely a contributing factor to the large gap in detections between a few $1000 \mathrm{~s}$ and $10^{4} \mathrm{~s}$ duration bursts.

GRBs 971208A, 060218A, 060814B, and 100316D all show the simplest $\gamma$-ray light curve, one broad or FRED-like pulse lasting anywhere from 1300 to 3000 s. Slightly more complicated, with a broad $\sim 200 \mathrm{~s}$ initial peak followed by a $1000 \mathrm{~s}$ tail, is the earliest documented ultra-long GRB discussed in the literature, GRB 840304A. Morphologically, FRED-like emission and the superposition of peaks together with longs tails is not uncommon. This behavior was seen by Giblin et al. (2002) in 40 BATSE GRBs and is common in many Swift-era bursts.

GRBs 090417B and 111209A show more complex and variable emission, with multiple overlapping peaks. The former had continuous emission, particularly in the softer BAT bands, for roughly $2000 \mathrm{~s}$, while the latter emitted in $\gamma$-rays for nearly $15 \mathrm{ks}$. GRB $101225 \mathrm{~A}$ is a weak burst detected over various Swift orbits with a redshift of $z=0.847$ assigned from the spectrum of a dim optical counterpart at the position of the GRB (Levan et al. 2013) and lower limits on the duration (>1650 s) and fluence (Racusin et al. 2011).

GRB 020410A has low-flux $\gamma$-ray emission and significant emission in X-rays, having been discovered by BeppoSAX (2-9 keV) and later in a ground analysis by $\mathrm{KW}$. Its $\gamma$-ray emission has various pulses and evidence of low-level excess in the softest band of KW out to $\sim 2500 \mathrm{~s}$. The pulses are temporally similar to bursts with interrupted emission and correspond with pulses observed by BeppoSAX, with interpulse periods lasting 50-100 s. This near-background interpulse period is likely not an indication of central-engine quiescence but a detectability problem, since this burst has a flux near the threshold of KW and has corresponding structure in the X-ray band. It is then possible that this characteristic of continued central-engine activity extends to bursts with interrupted emission, like our analysis of GRB 091024A indicates, and highlights the likely overlap of both categories.

In addition to the prompt $\gamma$-rays, about half of these bursts also have multi-wavelength afterglows. GRBs 971208A and 060814B do not have detected afterglows and apart from their duration, have no indication (e.g., spectra) that they are intrinsically different from other GRBs (Giblin et al. 2002; Pal'shin et al. 2008). GRB 090417B is an optically dark burst with a canonical X-ray light curve (Zhang et al. 2006), and GRB $111209 \mathrm{~A}$ is a seemingly extreme version of the former. The long $\gamma$-ray and X-ray emission of GRB 111209A lasts about $20 \mathrm{ks}$ and has been interpreted as emission from the collapse of a blue supergiant, while the late-time optical afterglow of GRB 111209A has been interpreted within the context of the external-shock model as emission from the external shock/FS or possibly the external shock/RS (Gendre et al. 2013; Stratta et al. 2013). GRB 101225A also shows long-lived X-ray and optical/infrared emission together with its weak $\gamma$-rays.

With different behavior from the rest, GRBs 060218A and $100316 \mathrm{D}$ are nearby bursts that have long-lived X-ray emission 
with thermal components ${ }^{23}$ and very low $E_{\text {peak }}$ values (Liang et al. 2006b; Starling et al. 2011). Various theoretical interpretations have been invoked to explain X-ray flashes and soft events, including off-axis viewing angle (Liang et al. 2006b; Guidorzi et al. 2009), shock breakout emission (Liang et al. 2006b; Nakar \& Sari 2012; Bromberg et al. 2011), and sub-energetic explosions (Soderberg et al. 2006, Margutti et al. 2013b). Regardless of the interpretation, the observations seem to imply that events similar to these GRBs are set apart in their prompt and afterglow properties and there is mounting evidence that they may require a different description within the framework of classical GRBs.

\section{DISCUSSION}

We have presented a multi-faceted analysis GRB 091024A as an example of an ultra-long GRB, and in the context of the sample of other such objects in order to shed light onto the population as a whole. This burst falls into a general category of bursts that show multiple episodes of $\gamma$-rays separated by large periods of quiescence or low-level emission. By performing a power-spectrum analysis we have shown that these episodes exhibit similar variability timescales, firmly tying them to similar central-engine activity. Spectrally, the episodes do not show extraordinary activity compared to other GRBs, the KW data revealing $E_{\text {peak }}$ values $\sim 300 \mathrm{keV}$, mild spectral evolution, and lower hardness ratio in later emission episodes.

The well-sampled early optical afterglow displays three bright peaks beginning just after the first $\gamma$-ray episode. These peaks are not coincident and show no correlation with the $\gamma$-ray peaks, nor do they show tracking behavior like the "naked-eye burst" GRB 080319B (Racusin et al. 2008), which would imply an internal origin. Since the first optical peak begins before the final $\gamma$-ray episodes it is natural to link it to the first episode. Our detailed afterglow modeling shows that this burst can be well modeled by a peak caused by a highly magnetized RS, followed by the onset of the FS with added energy injection. Since GRB 091024A has similar spectral characteristics as other GRBs with shorter interpulse emission and has an afterglow that can be reasonably well explained within the framework of a magnetized RS, we argue that this GRB is not fundamentally different but likely an event in the tail of the distribution of "classical" long GRBs. Whether or not this and other bursts with very long gamma-ray duration are a separate population is a relatively new topic recently brought to light by discussions on the observations of GRB 111209A (Gendre et al. 2013; Stratta et al. 2013) and 101225A (Levan et al. 2013).

\subsection{Statistical test}

We now examine the probabilities of discovering the number of observed events within the assumption of a log-normal distribution of durations to test whether the current observations require the definition of a distinct new class of GRBs. For reference, we show the one-dimensional duration, onedimensional fluence, and two-dimensional fluence-duration and flux-duration distributions in Figure 10. We selected the subset of the 591 GRBs detected by Swift with $\log$ (duration) $>0.7$ $(\gtrsim 5 \mathrm{~s})$ to avoid contamination from short bursts and fit this with a normal distribution (mean $=1.67, \sigma=0.51$ ). We then performed a $\chi^{2}$ test with the expected normal distribution, yielding a $\chi^{2}$ of 20.9 for 11 degrees of freedom, which corresponds to

\footnotetext{
23 In a reanalysis of the XRT data, Margutti et al. (2013b) show that the significance of this thermal component is substantially reduced.
}

a probability of accepting the null hypothesis of normality to $3.4 \%$. Truncation of the normal distribution at short durations may contribute to the deviation from normality, and performing this analysis with a truncated normal distribution having similar parameters yields slightly higher probabilities $\left(\chi^{2}=19.4\right.$, $P=5.4 \%$ ). The largest deviations from the expected distribution as probed by the $\chi^{2}$ test, however, are not from the bin containing the longest GRBs. The expected number of GRBs with duration longer than $\sim 630 \mathrm{~s}$ is approximately 9.75 events, similar to the 11 Swift-observed events. Reducing the total to nine bursts, simulating the absence of GRB 060218A and 100316D from the sample, does not significantly change the fit or probabilities. Only increasing our sample of observed bursts will allow us to fully probe this end of the distribution with a higher resolution. Since we are not able to robustly reject the simple null hypothesis of normality, this test is suggestive that, from the properties of the duration distribution, the observed events are sampled from the tail of the distribution and that there is no current justification for the more complex hypothesis of a separate population (i.e., Occam's razor).

\subsection{Progenitor models}

Large-mass progenitors have been recently invoked to produce a variety of interesting long-lived structures in $\gamma$-ray and X-ray-rich bursts (e.g., Peng et al. 2013, Nakauchi et al. 2013), but the relationship between the amount of material present for accretion, the unknown mechanism to start and stop the accretion flow, and how these scale with stellar mass and composition of the progenitor star are not well constrained. Lazzati et al. $(2010,2012)$ also caution on the difficulty of associating a central-engine activity with the $T_{90}$ duration of a given GRB and the possible effects of viewing angle on the ultimate duration of the burst. As an example of an alternative model to a significantly larger progenitor star, Wu et al. (2013) propose a fall-back accretion model interpretation (e.g., Kumar et al. 2008a, 2008b) for this type of long-lived central-engine activity. Observationally, X-ray rich bursts that imply long-lived central-engine activity appear to exhibit many similar qualities as "normal" GRBs (Feroci et al. 2001; Nicastro et al. 2004; in't Zand et al. 2004).

In a recent review, Woosley (2011) and Woosley \& Heger (2012) have theorized that the outer layers of a star may have sufficient angular momentum to form a disk, which would appear similar to a GRB jet, but longer and fainter provided the mass-loss rate is not high. They predict durations of $10^{4}-10^{5} \mathrm{~s}$ for both supergiant star and Wolf-Rayet binary progenitors, attributing GRB 101225 and similar bursts (i.e., 111209A) to one of these scenarios. Other studies examine the possibility that at least some GRBs are produced from binary pairs (e.g., Podsiadlowski 2007; Podsiadlowski et al. 2010), providing a viable alternative scenario to the blue supergiant hypothesis. The requirement that the jet fully penetrate the envelope of the star has proven challenging to models with large progenitors (Matzner 2003), but recent simulations indicate that this may be possible under certain conditions (e.g., Suwa \& Ioka 2011; Nakauchi et al. 2012, 2013). Conversely, Nakauchi et al. (2013) also find that for different values of the (highly uncertain) jet efficiency, wider weaker jets ( $>18-24$ degrees) have difficulty breaking out of the stellar envelope for several of their blue supergiant models, adding further constraints to this progenitor model. This becomes particularly important for bursts such as GRB 111209A whose derived jet opening angle is about $23^{\circ}$ (Stratta et al. 2013). 


\section{CONCLUSIONS}

The broadband data on GRB 091024A have provided a rich and detailed test of the underlying central engine that powers these interesting and energetic phenomena. Our conclusions are summarized as follows.

1. GRB 091024A has $\gamma$-ray emission lasting for about $1200 \mathrm{~s}$ that is separated into three separate emission episodes, with weak interpulse emission detected between the first and second episodes.

2. We report the spectroscopic redshift of this burst as $z=$ $1.0924 \pm 0.0002$.

3. The rich optical data set has three peaks that are not coincident in time with the gamma-ray emission. We interpret these peaks as emission from a highly magnetized external/RS in an intermediate shell thickness regime followed by the FS peak and rebrightening feature.

4. GRB 091024 shares many properties with "classical" GRBs and is likely an event in the tail of the distribution of long GRBs.

5. We analyze a sample of ultra-long GRBs discovered to date. These show natural variety in their characteristics and can be broadly described in two categories: bursts with interrupted and continuous $\gamma$-ray emission. With the likely exception of GRBs 060218A and 100316D, the properties of this sample do not yet provide a strong statistical motivation for defining a new, distinct population of GRBs and instead suggest ultra-long GRBs represent the tail of the duration distribution of the long GRB population.

Further diversified observations at many wavelengths and timescales are essential to further understand the nature of these enigmatic events.

F.J.V. acknowledges support from the UK Science and Technology Facilities Council. C.G.M. acknowledges funding from the Royal Society, the Wolfson Foundation, and the UK Science and Technology Facilities Council. We are grateful for excellent staff assistance at the various observatories where we obtained data. The Liverpool Telescope is operated by Liverpool John Moores University at the Observatorio del Roque de los Muchachos of the Instituto de Astrofísica de Canarias. The Faulkes Telescopes, now owned by the Las Cumbres Observatory Global Telescope network, are operated with support from the Dill Faulkes Educational Trust. KAIT and its ongoing operation were made possible by donations from Sun Microsystems, Inc., the Hewlett-Packard Company, AutoScope Corporation, Lick Observatory, the NSF, the University of California, the Sylvia and Jim Katzman Foundation, and the TABASGO Foundation. Some of the data presented herein were obtained at the W. M. Keck Observatory, which is operated as a scientific partnership among the California Institute of Technology, the University of California, and NASA; the Observatory was made possible by the generous financial support of the W. M. Keck Foundation. The Konus-Wind experiment is supported by a Russian Space Agency contract and RFBR grant 12-0200032-a. Swift, launched in 2004 November, is a NASA mission in partnership with the Italian Space Agency and the UK Space Agency. A.V.F.'s group at UC Berkeley has received generous financial assistance from Gary and Cynthia Bengier, the Christopher R. Redlich Fund, the Richard and Rhoda Goldman Fund, the TABASGO Foundation, NSF grant AST-1211916, and NASA/Swift grants NNX10AI21G and NNX12AD73G.
This work made use of data supplied by the UK Swift Science Data Centre at the University of Leicester.

\section{APPENDIX \\ ULTRA-LONG GAMMA-RAY BURSTS}

Below we detail the observations of the ultra-long GRBs with $\gamma$-ray durations longer than $\sim 1000$ s discussed in the literature. In parentheses we denote how we classified each burst, either as having continuous or interrupted $\gamma$-ray emission. Some of this information is summarized in Table 6.

\section{A1. GRB 840304A (Continuous)}

GRB 840304A is the earliest documented ultra-long GRB reported in the literature (Klebesadel et al. 1984). It was detected by Pioneer Venus Orbiter, International Cometary Explorer, and Vela 5B and its light curve consists of a two broad peaks lasting $\sim 200 \mathrm{~s}$ followed by a $1000 \mathrm{~s}$ tail. The fluence is $2.8 \times 10^{-3} \mathrm{erg} \mathrm{cm}^{-2}$. No afterglow is detected.

\section{A2. GRB 971208A (Continuous)}

Detected by CGRO/BATSE (Connaughton et al. 1997; Giblin et al. 2002), KW (Pal'shin et al. 2008), and BeppoSAX (Frontera et al. 2009; Guidorzi et al. 2011b), the light curve of this burst is one extremely bright and long-lasting FRED-like pulse lasting $2500 \mathrm{~s}$ and with a fluence of $2.55 \pm 0.11 \mathrm{erg} \mathrm{cm}^{-2}$ in the 15-1000 keV band (Pal'shin et al. 2008). This long pulse can be separated into a strong initial pulse that contains a large fraction of the fluence, followed by a long-lasting and low-level tail, similar to GRBs 840304A and 060814B. This behavior was seen by Giblin et al. (2002) in 40 BATSE GRBs and is also common in many Swift-era bursts. The spectrum shows hard to soft spectral evolution as a function of time, again similar to GRB 060418B, and the time-integrated $E_{\text {peak }}$ (over the entire burst interval) is a typical $144 \pm 12 \mathrm{keV}$. The spectrum of the intense initial peak (485 s) is slightly harder with a peak energy of $165 \pm 7 \mathrm{keV}$ (Pal'shin et al. 2008).

\section{A3. GRB 020410A (Continuous)}

This burst was detected by BeppoSAX in X-rays and as an offline detection by KW (Nicastro et al. 2004) and has four overlapping peaks lasting $>1300 \mathrm{~s}(2-10 \mathrm{keV})$ and fluence $>4.7 \times 10^{-6} \mathrm{ergcm}^{-2}$. The duration of the X-rays is only an upper limit as Earth occultation caused the observations to cease, but a reconstruction of the partially observed final peak and coincidence with the $\mathrm{KW}$ observations give an estimate of $\sim 1550 \mathrm{~s}$ for the duration and $2.8 \times 10^{-5} \mathrm{erg} \mathrm{cm}^{-2}$ for the $15-1000 \mathrm{keV}$ fluence of this burst, respectively. A possible $2.5 \sigma$ excess in the softer channels from $T_{0}+1500$ to $T_{0}+2500 \mathrm{~s}$ is also reported. This burst shows clear overlap of structure in the $\gamma$-rays and long X-ray emission, showing it is plausible that the $\mathrm{X}$-ray structure in other long-lived X-ray-rich bursts with shorter $\gamma$-ray durations (in 't Zand et al. 2004) could in fact be caused by a process similar to that which creates the weak $\gamma$-rays. No redshift is found for this burst but it is estimated as $0.9<z<1.5$ from the Amati relation (Amati et al. 2002). Late-time optical emission is detected and can be possibly explained by refreshed shocks or the emergence of a supernova (SN) component (Levan et al. 2005). By assuming this emission is from an SN, Levan et al. (2005) estimate the redshift as $z \sim 0.5$. No radio afterglow was detected to $>120 \mu \mathrm{Jy}$ at $8 \mathrm{GHz}$. 


\section{A4. GRB 060218A (Continuous)}

This burst is very similar to the description of GRB 100316D (See Section A.9 for further discussion). Its emission lasts for approximately $2100 \mathrm{~s}$ in the $S w i f t /$ BAT band, has an $E_{\text {peak }}=$ $4.9_{-0.3}^{+0.4} \mathrm{keV}$, and shows indications of a thermal component (Soderberg et al. 2006; Campana et al. 2006; Liang et al. 2006b). The fluence is $1.7 \times 10^{-5} \mathrm{erg} \mathrm{cm}^{-2}$ in the $15-150 \mathrm{keV}$ and corresponds to an $E_{\text {iso }}$ of $(6.2 \pm 0.3) \times 10^{49}$ erg (Campana et al. 2006; Soderberg et al. 2006) at $z=0.0331$ (Mirabal et al. 2006). The optical light curve is consistent shows two prominent peaks, the latter being consistent with the emergence of a SN (Campana et al. 2006). Radio observations are indicative of a mildly relativistic explosion in a quasi-spherical flow, with a jet opening angle of $>80^{\circ}$ and Lorentz factor of 2.3 at about five days (Soderberg et al. 2006).

\section{A5. GRB 060814B (Continuous)}

Showing similarity to GRB 971208A, the light curve of this burst shows a single FRED-like peak lasting about $2700 \mathrm{~s}$, with the most intense portion of the peak lasting $\sim 700 \mathrm{~s}$. This burst was detected in its entirety by KW and the initial portion by Ulysses, Mars Odyssey (HEND), Suzaku-WAM, and INTEGRAL-SPI-ACS (Pal'shin et al. 2008). Continued lowlevel emission that is likely associated with this burst was also detected in the softest KW channels, lasting on the order of $10^{4} \mathrm{~s}$. The spectrum shows hard-to-soft evolution and has typical peak energies, $374 \pm 30 \mathrm{keV}$ for the initial pulse and $341 \pm 61 \mathrm{keV}$ for the entire $2700 \mathrm{~s}$ pulse duration, giving a derived fluence of $(2.35 \pm 0.22) \times 10^{-4} \mathrm{erg} \mathrm{cm}^{-2}$ in the $18-1170 \mathrm{keV}$ range (Pal'shin et al. 2008).

\section{A6. GRB 080407A (Interrupted)}

This burst is an example of a burst with interrupted emission detected by $\mathrm{KW}$, showing two separate emission episodes lasting $\sim 160 \mathrm{~s}$ and $\sim 400 \mathrm{~s}$, respectively, separated by $\sim 1500 \mathrm{~s}$ (Pal'shin et al. 2012). Portions of this burst were detected by a variety of other spacecraft (see Pal'shin et al. 2012 and references therein). The first episode shows some sub-structure, with a hard and bright spike $\left(E_{\text {peak }}=325_{-25}^{+29} \mathrm{keV}\right)$ followed by a softer peak of similar duration $\left(E_{\text {peak }}=114_{-44}^{+77} \mathrm{keV}\right)$, and a total derived fluence of $(1.43 \pm 0.04) \times 10^{-4} \mathrm{erg} \mathrm{cm}^{-2}$ (20-1000 keV). The second episode, unfortunately, does not have spectral information but has an estimated fluence of $\sim 3 \times 10^{-4} \mathrm{erg} \mathrm{cm}^{-2}$, bringing the total fluence of this burst to approximately $4.4 \times 10^{-4} \mathrm{erg} \mathrm{cm}^{-2}(20-1000 \mathrm{keV}$; Pal'shin et al. 2012).

\section{A7. GRB 090417B (Continuous)}

Detected by Swift, this burst has four broad, overlapping peaks spanning $2130 \mathrm{~s}$ in the BAT $15-150 \mathrm{keV}$ band. This duration, however, is an upper limit as observations were stopped because of an earth limb constraint (Holland et al. 2010). Apart from its long duration, this burst is an example of a typical GRB, with a power law spectrum in the BAT band (fluence $>8.2_{-2.1}^{+1.0} \times 10^{-6} \mathrm{erg} \mathrm{cm}^{-2}$ ) and a doubly broken power law X-ray afterglow with some flaring activity. The $E_{\text {peak }}$ is estimated as $>150 \mathrm{keV}$ from the BAT data. This burst is also dark in UV, optical, and infrared wavelengths with line-of-sight dust extinction as the likely cause. A host-galaxy candidate has been identified and the presumptive redshift for this burst is $z=0.345$, giving an upper limit of $\gtrsim 6.3 \times 10^{51} \mathrm{erg}$ for the isotropic equivalent energy.

\section{A8. GRB 091024A (Interrupted)}

See the main text.

\section{A9. GRB 100316D}

This local event $(z=0.0591$; Chornock et al. 2010; Starling et al. 2011) shows long-lasting and smooth $\gamma$-ray emission, lasting approximately $1300 \mathrm{~s}$. The very soft spectrum peaks at $\sim 10-42 \mathrm{keV}$ and shows possible evidence of a thermal component in the X-ray emission, similar to GRB 060218A (Starling et al. 2011). The fluence of $(5.1 \pm 0.39) \times 10^{-6} \mathrm{erg} \mathrm{cm}^{-2}$ (15-150 keV) coupled with the low redshift implies an isotropic equivalent energy of approximately $3.9 \times 10^{49} \mathrm{erg}$ (Starling et al. 2011). The optical light curve is also very structured and the late-time emission has been associated with SN emission (Wiersema et al. 2010).

Recent work by Margutti et al. (2013b) indicates that the thermal component is likely less significant that previously thought and reports a significant soft X-ray excess at late times $(>10$ days). In addition, they provide radio observations that infer the $\mathrm{KE}$ coupled to the non-relativistic material of $E_{k} \sim 10^{52} \mathrm{erg}$ and about $10^{49}$ erg coupled with a mildly relativistic ejecta with large opening angle $\left(>80^{\circ}\right)$. Various theoretical interpretations have been put forward (see Section 5.2) but in general there is growing support that events such as GRB 100316D and 060218A are related to a $\mathrm{BH}$-torus or magnetar system like "classical" GRBs, but more abundant and significantly less energetic and collimated than their more distant counterparts.

\section{A10. GRB 101225A (Continuous)}

This weak burst detected over various Swift orbits has both prompt and afterglow emission detected by all three instruments aboard Swift and ground-based facilities (Racusin et al. 2011; Campana et al. 2011; Thöne et al. 2011). Due to gaps in the data, the duration and fluence are upper limits of $\gtrsim 1650 \mathrm{~s}$ and $3 \times 10^{-6} \mathrm{erg} \mathrm{cm}^{-2}$ in the $15-150 \mathrm{keV}$ band (Racusin et al. 2011; Campana et al. 2011). The X-ray light curve is very extended and variable and has a shallow decay followed by a steep decay beginning at $\sim 21 \mathrm{ks}$. The optical afterglow also shows variability and a gradual decline until the emergence of a likely SN bump beginning after approximately 10 days (Thöne et al. 2011).

Initially attributed to the accretion of a minor body onto a compact object at Galactic distances (Campana et al. 2011), or the merger of a helium star and neutron star (NS) at $z \approx 0.33$ (Thöne et al. 2011). This redshift was obtained by fitting of an SN template to the late-time optical data. These scenarios have recently been questioned by the identification of the redshift of this burst at $z=0.847$ by Levan et al. (2013) from a faint but coincident optical counterpart, which significantly increases the energy budget. The burst properties (fluence, duration) are upper limits, as the event was already in progress when it entered the Swift field of view (Racusin et al. 2011) and the true duration could possibly surpass $10^{4} \mathrm{~s}$ (Campana et al. 2011; Levan et al. 2013).

\section{A11. GRB 110709B (Interrupted)}

Similar to GRB 091024A, this burst has two large emission episodes lastin $55.6 \pm 3.2 \mathrm{~s}$ and $259.2 \pm 8.8 \mathrm{~s}$, separated by $\sim 11$ minutes of quiescence. This interesting event triggered Swift on both of its episodes and lasts about $900 \mathrm{~s}$ in the 15-150 keV band (Zhang et al. 2012). A detailed spectral 
analysis with simultaneous KW data shows hard-to-soft spectral evolution and typical GRB energies across the two pulses: $E_{\text {peak }}=311_{-38}^{+45} \mathrm{keV}$ and $116_{-8}^{+9} \mathrm{keV}$, respectively. This corresponds to $8.95_{-0.62}^{+0.29} \times 10^{-6}$ and $1.34_{-0.07}^{+0.05} \times 10^{-5} \mathrm{erg} \mathrm{cm}^{-2}$. This is a an optically dark burst with no redshift determination. It has been interpreted as a so-called "double-burst" caused by a two-step collapse to a NS then a BH, which creates the two intense emission episodes (Zhang et al. 2012).

\section{A12. GRB 111209A (Continuous)}

This very weak but continuous burst has combined BAT and $\mathrm{KW}$ emission lasting $\sim 15 \mathrm{ks}$ with $E_{\text {peak }}=310 \pm 53$ corresponding to about $(4.9 \pm 0.61) \times 10^{-4} \mathrm{erg} \mathrm{cm}^{-2}$. The redshift has been identified as $z=0.677$, implying an isotropic equivalent energy of $(5.8 \pm 0.73) \times 10^{53} \mathrm{erg}$ (Gendre et al. 2013).

The seemingly canonical X-ray light curve (Zhang et al. 2006) has a shallow decay (slope $=0.544 \pm 0.003$ ) before breaking at about $20 \mathrm{ks}$ and exhibiting typical steep decay, plateau, and normal decay behavior (Gendre et al. 2013). Although rich in structure and complexity, the X-ray and optical afterglows can be well described within the framework of the external-shock model (Stratta et al. 2013). There is late-time rebrightening in the optical light curve that is possibly indicative of SN emission, but this remains inconclusive. In the literature, this burst has been interpreted as the collapse of a blue supergiant star with a possible binary companion (Gendre et al. 2013; Stratta et al. 2013).

\section{REFERENCES}

Akerlof, C., Balsano, R., Barthelmy, S., et al. 1999, Natur, 398, 400 Amati, L., Frontera, F., Tavani, M., et al. 2002, A\&A, 390, 81 Arnold, D. M., Steele, I. A., Bates, S. D., Mottram, C. J., \& Smith, R. J. 2012, Proc. SPIE, 8846, 2

Band, D., Matteson, J., Ford, L., et al. 1993, ApJ, 413, 281

Berger, E., Kulkarni, S. R., \& Frail, D. A. 2003, ApJ, 590, 379

Bissaldi, E., \& Connaughton, V. 2009, GCN Circ., 10070, 1

Boër, M., Atteia, J. L., Damerdji, Y., et al. 2006, ApJL, 638, L71

Bromberg, O., Nakar, E., \& Piran, T. 2011, ApJ, 739, 55

Burrows, D. N., Romano, P., Falcone, A., et al. 2005, Sci, 309, 1833

Camapana, S., Lodato, G., D’Avanzo, P., et al. 2011, Natur, 480, 69

Campana, S., Mangano, V., Blustin, A. J., et al. 2006, Natur, 442, 1008

Cano, Z., Guidorzi, C., Mundell, C. G., et al. 2009, GCN Circ., 10066, 1

Chincarini, G., Mao, J., Margutti, R., et al. 2010, MNRAS, 406, 2113

Chornock, R., Berger, E., Levesque, E. M., et al. 2010, arXiv:1004.2262

Chornock, R., Li, W., \& Filippenko, A. V. 2009, GCN Circ., 10075, 1

Connaughton, V., Kippen, R. M., Preece, R., \& Hurley, K. 1997, IAUC, 6785, 1

Drago, A., \& Pagliara, G. 2007, ApJ, 665, 1227

Drago, A., Pagliara, G., \& Schaffner-Bielich, J. 2008, J. Ph. G. Nucl. Part. Phys., 35, 014052

Evans, P. A., Beardmore, A. P., Page, K. L., et al. 2007, A\&A, 469, 379

Evans, P. A., Beardmore, A. P., Page, K. L., et al. 2009, MNRAS, 397, 1177

Fan, Y. Z., \& Wei, D. M. 2005, MNRAS, 364, L42

Fenimore, E. E., \& Ramirez-Ruiz, E. 2000, arXiv:astro-ph/0004176

Feroci, M., Antonelli, L. A., Soffitta, P., et al. 2001, A\&A, 378, 441

Filippenko, A. V., Li, W. D., Treffers, R. R., \& Modjaz, M. 2001, in ASP Conf.

Ser. 246, Small-Telescope Astronomy on Global Scales, ed. B. Paczyński, W.-P. Chen, \& C. Lemme (San Francisco, CA: ASP), 121

Fishman, G. J., Meegan, C. A., Wilson, R. B., et al. 1993, A\&AS, 97, 17

Freedman, D. L., \& Waxman, E. 2001, ApJ, 547, 922

Frontera, F., Guidorzi, C., Montanari, E., et al. 2009, ApJS, 180, 192

Gehrels, N., Chincarini, G., Giommi, P., et al. 2004, ApJ, 611, 1005

Gendre, B., Klotz, A., Palazzi, E., et al. 2010, MNRAS, 405, 2372

Gendre, B., Stratta, G., Atteia, J. L., et al. 2013, ApJ, 766, 30

Giblin, T. W., Connaughton, V., van Paradijs, J., et al. 2002, ApJ, 570, 573

Golenetskii, R., Aptekar, R., Mazets, E., et al. 2009, GCN Circ., 10083, 1

Gomboc, A., Kobayashi, S., Guidorzi, C., et al. 2008, ApJ, 687, 443

Gruber, D., Krhler, T., Foley, S., et al. 2011, A\&A, 528, A15

Guidorzi, C., Clemens, C., Kobayashi, S., et al. 2009, A\&A, 499, 439

Guidorzi, C., Frontera, F., Montanari, E., et al. 2006a, MNRAS, 371, 843
Guidorzi, C., Kobayashi, S., Perley, D. A., et al. 2011a, MNRAS, 417, 2124

Guidorzi, C., Lacapra, M., Frontera, F., et al. 2011b, A\&A, 526, A49

Guidorzi, C., Monfardini, A., Gomboc, A., et al. 2006b, PASP, 118, 288

Harrison, R., \& Kobayashi, S. 2013, ApJ, 772, 101

Henden, A., Gross, J., Denny, B., Terrell, D., \& Conney, W. 2009, GCN Circ., 10073,1

Holland, S. T. 2010, ApJ, 717, 223

Horne, K. 1986, PASP, 98, 609

in 't Zand, J. J. M., Heise, J., Kippen, R. M., et al. 2004, in ASP Conf. Ser. 312, Third Rome Workshop on Gamma-Ray Bursts in the Afterglow Era, ed. M. Feroci, F. Frontera, N. Masetti, \& L. Piro (San Francisco, CA: ASP), 18

Kalberla, P. M., Burton, W. B., Hartmann, D., et al. 2005, A\&A, 440, 775

Klebesadel, R. W., Laros, J. G., \& Fenimore, E. E. 1984, BAAS, 16, 1016

Klebesadel, R. W., Strong, I. B., \& Olson, R. A. 1973, ApJL, 182, L85

Kobayashi, S., Piran, T., \& Sari, R. 1997, ApJ, 490, 92

Kobayashi, S., \& Sari, R. 2000, ApJ, 542, 819

Kobayashi, S., \& Zhang, B. 2003, ApJL, 582, L75

Kopač, D., Kobayashi, S., Gomboc, A., et al. 2013, ApJ, 772, 73

Kouveliotou, C., Meegan, C. A., Fishman, G. J., et al. 1993, ApJL, 413, L101

Kulkarni, S., Frail, D. A., Sari, R., et al. 1999, ApJ, 522, 97

Kumar, P., Narayan, R., \& Johnson, J. L. 2008a, MNRAS, 388, 1729

Kumar, P., Narayan, R., \& Johnson, J. L. 2008b, Sci, 321, 376

Laskar, T., Berger, E., Zauderer, B. A., et al. 2013, arXiv:1305.2453

Lazzati, D., Morsony, B. J., \& Begelman, M. C. 2010, ApJL, 700, L47

Lazzati, D., Morsony, B. J., Blackwell, C. H., \& Begelman, M. C. 2012, ApJ, 750, 68

Levan, A., Nugent, P., Fruchter, A., et al. 2005, ApJ, 624, 880

Levan, A., Tanvir, N. R., Starling, R. L. C., et al. 2013, arXiv:1302.2352

Li, T.-P. 2001, ChJAA, 1, 313

Li, T. P., \& Muraki, Y. 2001, ApJ, 578, 374

Li, W., Filippenko, A. V., Chornock, R., \& Jha, S. 2003, PASP, 115, 844

Liang, E. W., Li, L., Gao, H., et al. 2013, ApJ, 774, 13

Liang, E. W., Zhang, B., O’Brien, P. T., et al. 2006a, ApJ, 646, 351

Liang, E. W., Zhang, B. B., Stamatikos, M., et al. 2006b, ApJL, 653, L81

Lloyd-Ronning, N. M., \& Zhang, B. 2004, ApJ, 613, 477

López-Cámara, D., Lee, W. H., \& Ramirez-Ruiz, E. 2010, ApJ, 716, 1308

Margutti, R. 2009, PhD Thesis, Università degli Studi di Milano-Bicocca, http://boa.unimib.it/handle/10281/7465

Margutti, R., Bernardini, G., Barniol-Duran, R., et al. 2011, MNRAS, 410, 1064

Margutti, R., Guidorzi, C., Chincarini, G., et al. 2008, in AIP Conf. Proc. 1065, 2008 Nanjing Gamma-ray BURST Conference, ed. Y.-F. Huang, Z.-G. Dai, \& B. Zhang (Melville, NY: AIP), 259

Margutti, R., Guidorzi, C., Chincarini, G., et al. 2010, MNRAS, 406, 2149

Margutti, R., Soderberg, A. M., Wieringa, M. H., et al. 2013b, arXiv:1308.1687

Margutti, R., Zaninoni, E., Bernardini, M. G., et al. 2013a, MNRAS, 428, 729

Marshall, F. E., Baumgartner, W. H., Beardmore, A. P., et al. 2009, GCN Circ., 10062, 1

Matheson, T., Filippenko, A. V., Ho, L. C., Barth, A. J., \& Leonard, D. C. 2000, AJ, 120, 1499

Matzner, C. D. 2003, MNRAS, 345, 575

Melandri, A., Guidorzi, C., Kobayashi, S., et al. 2009, MNRAS, 395, 1941

Melandri, A., Kobayashi, S., Mundell, C. G., et al. 2010, ApJ, 723, 1331

Melandri, A., Mundell, C. G., Kobayashi, S., et al. 2008, ApJ, 606, 1209

Mészáros, P., \& Rees, M. J. 1999, MNRAS, 306, 39

Mirabal, N., \& Halpern, J. P. 2006, GCN Circ., 4792, 1

Molinari, E., Vergani, S. D., Malesani, D., et al. 2007, A\&A, 469, 13

Morton, D. C. 1991, ApJS, 77, 119

Mundell, C. G., Cano, Z., Guidorzi, C., et al. 2009, GCN Circ., 10063, 1

Nakar, E., \& Piran, T. 2002, ApJ, 572, 139

Nakar, E., \& Piran, T. 2004, MNRAS, 353, 647

Nakar, E., \& Sari, R. 2012, ApJ, 747, 88

Nakauchi, D., Kashiyama, K., Suwa, Y., \& Nakamura, T. 2013, arXiv:1307.5061

Nakauchi, D., Suwa, Y., Sakamoto, T., Kashiyama, K., \& Nakamura, T. 2012, ApJ, 759, 128

Nicastro, L., in't Zand, J. J. M., Amati, L., et al. 2004, A\&A, 427, 445

Oke, J. B., Cohen, J. G., Carr, M., et al. 1995, PASP, 107, 375

Paciesas, W. S., Meegan, C. A., Pendleton, G. N., et al. 1999, ApJS, 122, 465

Page, K. L., \& Marshall, F. E. 2009, GCN Circ., 10069, 1

Page, K. L., Willingale, R., Osborne, J. P., et al. 2007, ApJ, 663, 1125

Pal'shin, V., Aptekar, R., Frederiks, D., et al. 2008, in AIP Conf. Proc. 1000,

Gamma-ray Bursts 2007: Proc. of the Santa Fe Conf., ed. M. Galassi, D.

Palmer, \& E. Fenimore (Melville, NY: AIP), 117

Pal'shin, V., et al. 2012, in Proc. Science Conf. Proc., Gamma-ray Burst 2012 Conference (GRB 2012) (Trieste, Italy: PoS), 40

Panaitescu, A., \& Kumar, P. 2001, ApJL, 560, L49

Park, H. S. 1997, Technical Report, Gamma-ray Bursts

Park, H. S., Williams, G. G., Hartmann, D. H., et al. 2002, ApJ, 571, 131 
Peng, F. K., Hu, Y. D., Xi, S. Q., et al. 2013, arXiv:1302.4876

Podsiadlowski, P. 2007, RSPTA, 365, 1163

Podsiadlowski, P., Ivanova, N., Justham, S., \& Rappaport, S. 2010, MNRAS, 406, 840

Qin, Y., Liang, E.-W., Liang, Y.-F., et al. 2013, ApJ, 763, 15

Quilligan, F., McBreen, B., Hanlon, L., et al. 2002, A\&A, 385, 377

Racusin, J., Cummings, J., Holland, S., et al. 2011, GCNR, 314.1, 1

Racusin, J. L., Karpov, S. V., Sokolowski, M., et al. 2008, Natur, 455, 183

Ramirez-Ruiz, E., Merloni, A., \& Rees, M. J. 2001, MNRAS, 324, 1147

Reichart, D. E., Lamb, D. Q., Fenimore, E. E., et al. 2001, ApJ, 552, 57

Sakamoto, T., Barthelmy, S. D., Baumgartner, W. H., et al. 2011, ApJS, 195, 2

Sari, R., \& Piran, T. 1999, ApJ, 520, 641

Soderberg, A. M., Kulkarni, S. R., Nakar, E., et al. 2006, Natur, 442, 1014

Starling, R. L. C., Wiersema, K., Levan, A. J., et al. 2011, MNRAS, 411, 2792

Steele, I. A., Mundell, C. G., Smith, R. J., Kobayashi, S., \& Guidorzi, C. 2009, Natur, 462, 767

Stratta, G., Gendre, B., Atteia, J. L., et al. 2013, arXiv:1306.1699

Suwa, Y., \& Ioka, K. 2011, ApJ, 726, 107

Thöne, C. C., de Ugarte Postigo, A., Fryer, C. L., et al. 2011, Natur, 480, 72

Tikhomirova, Y. Y., \& Stern, B. E. 2005, AstL, 31, 291
Updike, A. C., Hartmann, D. H., Milne, P. A., \& Williams, G. G. 2009, GCN Circ., 10074, 1

van Dokkum, P. G. 2001, PASP, 113, 1420

Vestrand, W. T., Wozniak, P. R., Wren, J. A., et al. 2005, Natur, 435, 178

Vestrand, W. T., Wren, J. A., Wozniak, P. R., et al. 2006, Natur, 442, 172

Virgili, F. J., Qin, Y., Zhang, B., \& Liang, E. W. 2012, MNRAS, 424, 2821

Wade, R. A., \& Horne, K. 1988, ApJ, 324, 411

Wei, D. M., Yan, T., \& Fan, Y. Z. 2006, ApJL, 636, L69

Wiersema, K., D'Avanzo, P., Levan, A. J., et al. 2010, GCN Circ., 10525, 1 Woosley, S. E. 2011, Gamma-Ray Bursts (Cambridge: Cambridge Univ. Press)

Woosley, S. E., \& Heger, A. 2012, ApJ, 752, 32

Wu, X. F., Hou, S. J., \& Lei, W. H. 2013, ApJL, 767, L36

Yamazaki, R., Toka, K., Ioka, K., \& Nakamura, T. 2006, MNRAS, 369, 311

Zhang, B., Fan, Y. Z., Dyks, Jaroslaw, et al. 2006, ApJ, 642, 354

Zhang, B., \& Kobayashi, S. 2005, ApJ, 628, 315

Zhang, B., Kobayashi, S., \& Mészáros, P. 2003, ApJ, 595, 950

Zhang, B., Liang, E., Page, K. L., et al. 2007, ApJ, 655, 989

Zhang, B., Zhang, B.-B., Virgili, F. J., et al. 2009a, ApJ, 703, 1696

Zhang, B. B., Burrows, D. N., Zhang, B., et al. 2012, ApJ, 748, 132

Zhang, B. B., Zhang, B., Liang, E. W., \& Wang, X. Y. 2009b, ApJL, 690, L10 